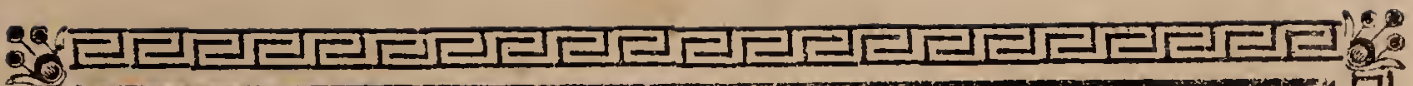

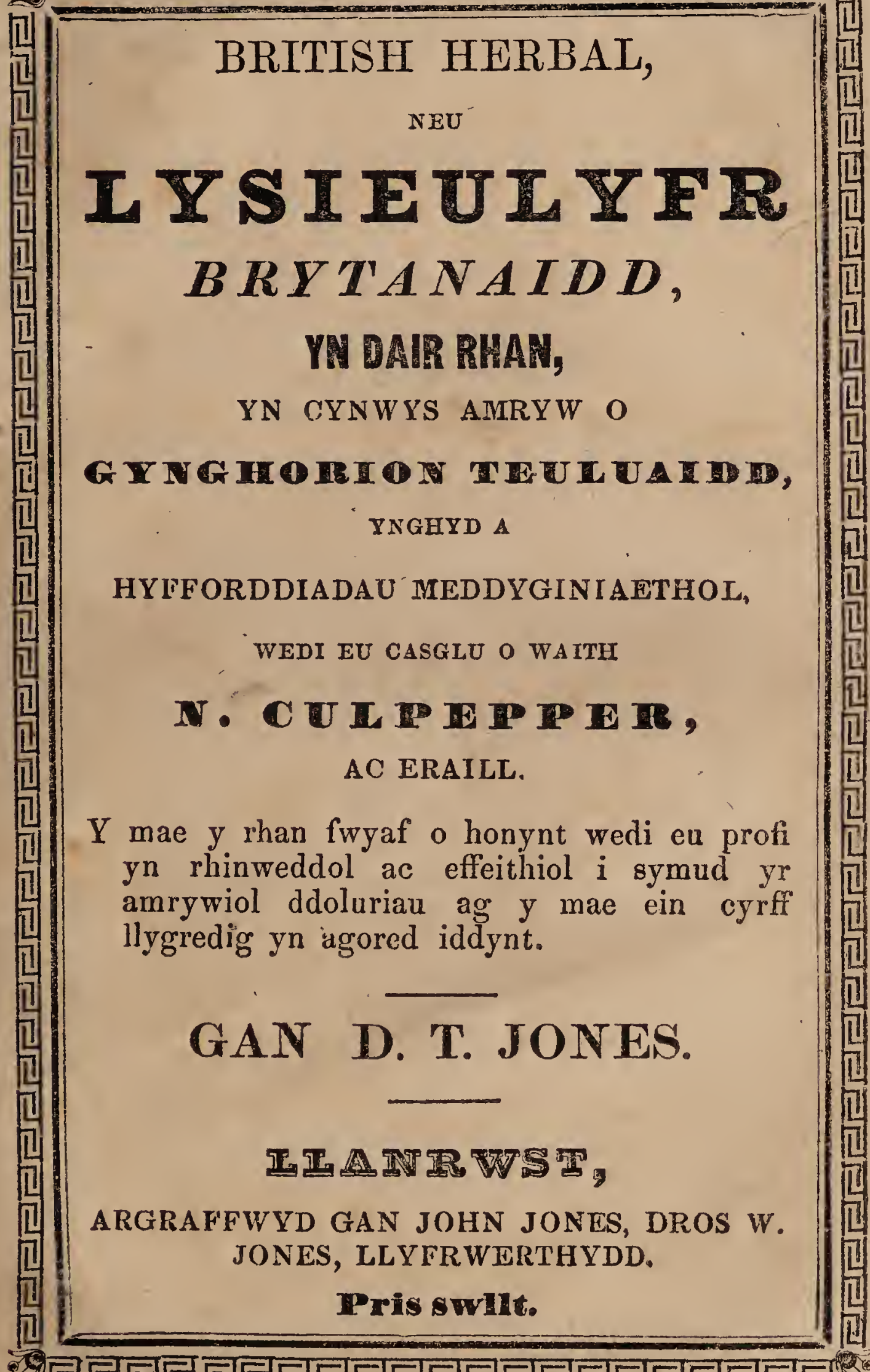


Supp A ICun

$$
6131210
$$




\section{BRITISH HERBAL,}

NEU

\section{LYSIEULYFR BRYTANAIDD,}

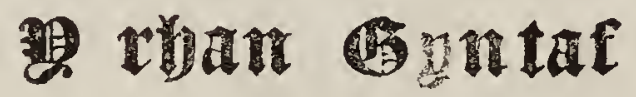

YN CYNWYS

900 GYNGHORION TEULUAIDD,

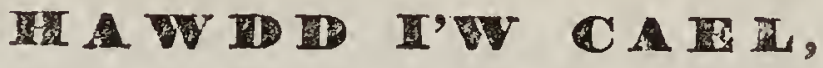

WRDI EU CASGLU O WAITH

N. CULPEPPER,

AC EREILL.

Y MAR'R RHAN FWYAF O'R CYNGHORION WEDI EU PROFI YN IHINWEDDOT AC EFFEITHIOL I SYMUD YR AMRYWIOL DDOLURIAU AG Y MAB EIN CYRFF LLYGREDIG YN DDAROSTYNGEDIG IDDYNT: AC $Y$ MARNT YN HOLLOL I'W DEFNYDDIO O DDAIL A LLYSIAU EIN GWLAD IIN HUNAIN.

HEFYD CYFARYYDOYD I OLLWNG GWAED, AC I GYMRRYD PURGL.

\section{GAN D.T. JONES.}

Y TRYOYBD ATRRAREIAD,

\section{政 Iancust:}

ARGRAFFWYD AC AR WERTH GAN J, JONIS. 



\section{AT Y DARLLENYDDION.}

YCHidig Phisygwriaeth deuluaidd, wedi eu defnyddio o ddail ein gerddi, ac amrywiol lysiau gwylltion y maesydd, fel ag y gallo pob dyn nad allo ond darllen Cymraeg yn unig, fod yn Physygwr iddo ei hun. Trwy fanwl chwilo y lly fr bychan hwn, gellwchugael cyfarwyddyd i symud ac i wellhau y rhan fwyaf o'r amrywiol ddoluriau ag y mae ein cyrph gwael yn ddarostyngedig iddynt.

Fe welodd yr Holl-wybodol a'r Galluog Dduw, o’i hunan-gynhyrfiol drugaredd a daioni iddyn pechadurus, fod yn dda $i$ blanu Balm y Feddyginiaeth yn ein gerddi, ac o flaen ein drysau; ac o ddeutu ein llwybrau, fel ag y gallom ninau, gyda chalon 
lawn o ddiolchgarwch, estyn allan eir dwylaw, a chymeryd yn feunyddiol o Falm y Feddyginiaeth mewn dydd o boen a gorthrymder corphorol.

Nid ydwyf ddim am eich gyru yn bresenol i'r India nac America i ymofyn meddyginiaeth, ond am droi eich wynebau i'ch gerddi a'ch maesydd eich hunain.

Y mae y Creawdwr mawr yn ei ddoeth drefn ei hun, wedi rhoddi amrywiol ddail a llysiau cyfaddas yn mhob gwlad tu ag at feddyginiaethu ei thrigolion: ond $y$ bai sydd arnom ein hunain am nad ydym yn eu deall. Trwm ydyw gweled dynion yn eu sathru dan draed fel pethau o ddim gwerth, er ei fod yn sicr y gallai un llysieuyn, ond ei iawn ddefnyddio, fod yn fwy ei werth i'r clwyfus nâ holl drysorau yr India. Am hyny da fyddai i ddynion ymdrechu llawer mwy atn adnabod en rhinwedd a'u heffaith yn fwy manwl. 
Ychydig gyfaruyddyd $i$ ollwng gwaed, a'r amser mwyaf' cyfaddas i fod yn ddiberygl.

Pan ddechreuwyd y gelfyddyd o waedu, mae yn ddiddadl ei bod o ddyben da, i symud ymaith ac i wrtawynebu llawe 0 ddoluriau; ac fe all eto ateb yr un dyben trwy ei iawn ddefnyddio yn ddeallus, ac nid yn fyrbwyll. 1, Y mae gwaedu ar y fraich chwith yn rhagorol o dda rhag dolur yn y pen; ac y mae hefyd yn dda yn yr un modd i'r rhai sydd wedi cael codymau. - Y mae rhai dysgedigion yn dal allan fod gwaedu yn hollawl feddyginiaeth i un-arhugain o ddoluriau, heb gynnorthwy un math o phisygwriaeth; ond y mae yn dda i lawer yn ychwaneg trwy gasglu phisygwriaeth $\mathrm{i}$ gyd-weithio $\hat{a} g$ ef.

Pan y byddo y lleuad ar eij chynydd, gallwch waedu yr amser a fynoch, y dydd a'r nôs; ond pan y byddo y lleuad ar gilio, nis gallwch waedu ond yn y bore yn unig. Gwaedu a ellir ei arferyd yn esmwyth o fis Mawrth hyd Dachwedd; ac na waedwch 
Adim o Ragfyr hyd ddiwedd Chwefror, os grallwch beidio. Misoedd Mawrth ao Ebrill, a Thachwedd, ydyw y tri goreu yn y flwyddyn i ollwng gwaed; ond fe ellir ei wneud yn ddiberygl o'r nawfed o Fawrth hyd y pedwerydd ar bymtheg o Dachwedd; - ond i ochelyd y perygl, peidied neb a mentro $\mathrm{i}$ agoryd gwythien ond $\mathrm{y}$ rhai a fyddant yn hyddysg ar y gwaith.

$Y$ mae tri math o bobl na ddylent ollwng gwaed ar neb; yn 1, y mae rhai sydd annealladwy, ac heb fod yn hyddysg ar y gwaith, ond eu bod yn pretendio yn unig; yn 2, y rhai sydd â golwg drwg ganddynt; ac yn 3 , y rhai sydd â dwylaw crynedig ganddynt. Ac yn olaf, na adewch i ferched waedu arnoch; na neb âg arfau rhydlyd. Ond hyn a ddywedaf wrthych cyn dibenu, os ewch at ddyn deallus, fe wyr pa wythien sydd i'w hagor at bob dolur; ac fe $\hat{w} y r$ hefyd pa faint o waed i'w ollwng wrth edrych ar y clwyfau. 


\section{HERBAL, \&c.}

Cyngor rhag y Darfodedigaeth, (Consumption) os cymerir mewn pryd.

CYMERWCH lonaid llaw o horownd, a haner hyny o riw'r gerddi, mewn dau chwart o ddwfr, a dau bwys o siwgr coch ynddo; a'u berwi yn nghyd nes wastio'r haner, a plottelwch; cymerwch o hono dair llonaid llwy fwrdd bob bore ar eich cythlwng.

\section{Rhag y Cryd.}

Cymerwch gamomile, a llysiau'r dryw, a chribau Sant Fraid, a dail neu wraidd carn yr ebol, a wraidd Eringo, neu gelyn y môr, a hops, yr un aint o bob un; a berwch hwynt oll mewn dwfr, a hoddwch ynddo ddigon o driagl, ac yfwch o hono an fyddoch yn gynhes yn y gwely; a chwyswch ldwy awr ar ei ol.

\section{I gryfhau'r Ystumog.}

Cymerwch peppermint, a chribau Sant Ffraid, a urans y gôg, yr un faint o bob un, a'u berwi yn ghyd mewn dwfr, a rhoddwch ynddo ychydig iwgr, neu ryw beth melus, ac yfwch o hono th eich rheswm. 
Rhag brathiad Ci cynddeiriog a Nadrodd.

Cymerwch fôm, a gwraidd cacamwcci, a mintys a hâd neu wraidd A lexander, a chribau Sant ffraid ac ysgaw Mai, a dail llyriaid, a rhiw'r gerddi cymerwch yr un faint o bob un, a berwch hw! mewn gwîn gwyn, yfwch y gwîn, a rhoddwch y daî yn allanol ar $\mathbf{y}$ lle.

\section{Rhag pocri Guaed.}

Cymerwch gribau Sant firaid, a dail nieri, rhoses cochion, a dail tafol cochion, yr un faint bob un, a'u berwi yn nghyd mewn gwîn coch; ( neb na allo gael gwin, rhaid iddo gymeryd dwfr) meluswch $\hat{a}$ siwgr gwyn, ac yfwch yn fynych , hono, ac jach a fyddwch.

\section{Rhag piso Gwaed.}

Cymerwch cwmphri a dail llyriaid, a smallage at yr un faint; a berwch yn nghyd, a rhoddwc ynddo haner pwys o siwgr gwyn i bob peint, $x$ yfwch o hono, ac iach a fyddwch.

Rhag y Pâs ar Blant.

Cymerwch ddail rhoccos, a theim y gerddi, theim gwyllt, berwch hwy yn nghyd, a meluswc â siwgr candi, a gadewch i'r plentyn yfed yn helaet o hono. 
Rhay y Consumsiwn, mewn hen ac icuangc.

Cymerwch wraidd cacamwcci, a gwr aidd dainty llew, a gwraidd carn yr ebol, a horownd, juniperberries, a resins yr haul, a berwch oll yn nghyd, a rhoddwch ynddo yn bur helaeth o siwgr coch, a berwch dros hir amser ar dân araf mewn llestr cauedig; cymerwch lonaid cwpan dê o hono yn dwymn dair gwaith yn y dydd.

\section{Rhag fitiau Confulsiwn.}

Cymerwch gribau Sant ffraid, penuyroyal, a dail 1. gwinwydd, at yr un faint o bob un, a berwch hwy mewn gwin a dwfr, ac yfwch o hono yn fynych, a byddwch iach.

\section{Rhag y Peswch.}

Cymerwch bay leaves, cribau Sant Ffraid, a swraidd neu flodau carn yr ebol, a hâd cywarch, fic isop, a dail tafod y ci, neu ddail tafol, a junipermerries, a gwallt y forwyn, a gwraidd rhoccos, a lail clust y llygoden, a mwstard gwyllt, rhosmari, saits; berwch at yr un faint o bob un, a'r rhai ydd gyfoethog gallant yn llwyddianus eu berwi newn gwin, ond y mae yn debyg y bydd raid i's ylodion eu berwi mewn dwfr, a'u melysn â mêl eu siwgr coch. Nid yw ond ofer rhoddi cyfaryddyd i'w gymeryd, yr ydwyf yn disgwyl y gwna ch deall naturiol eich cyfarwyddo, fel ag y byddo clwyf yn gofyn, a'ch nerth chwithau yn cynnal. 
Rhaig llosg tân.

Cymerwch beint o olew traed defaid, a phedair wns o read lead, ac un wns o gŵyr melyn; berwch ynghyd am dair awr, ar dân araf, gan ei droi, ac yna rhoddwch ato yn niwedd y berwi, haner wns o oil of elder, neu ysgaw, a gadewch iddo oeri, a thrusiwch y dolur âg ef unwaith ye y dydd.

\section{Un arall at yr un achos.}

Cymerwch ychydig lapis powdr, neu brown dust, (gellwch ei gael yn siop y Druggist), a chymysgwch mewn dwfr eira; gellwch wneuthur: digon yn y gauaf i barhau drwy'r flwyddyn; a chedwch at eich angen: mae yn diffodd $y$ tân, ac yn iachau y dolur.

\section{Un arall.}

Cymerwch linseed oil, a melyn ŵy, a siwgs coch, ac ychydig ddwfr, a chymysgwch yn dda hyd nes tewycho, a chymysgwch bob tro wrth ei arferyd.

\section{Un arall.}

Cymerwch spirit of wine, a champhor, a spiri of turpentine, ac oil of St. John, yr un faint o bol un ag eneiniwch y lle âg ef. Profwch ei fod yu dda $\mathrm{i}$ ddiffoddd tân.

\section{$Y$ ffordd $i$ wneuthur Dwfr Llygaid.}

Cymerwch haner peint o frandy, a dau beint. 
ddwfr ffynon oer, ac un whs o sugar of lead; a chymyrgwch ynghyd, a photelwch at eich achos.

\section{Un arall.}

Cymerwch sug celandine, a llygad y dydd, a dail llyriad, a llysiau'r esgyrn, a chymerwch yr un faint o bob un, 2 rhoddwch ynddo ychydig fêl, a rhoddwch o hono yn eich llygaid fel y byddo yr angen yn gofyn.

\section{I atal gwaed mewn briwiau.}

Cymerwch ledr wedi ei losgi a'i wneud yn bowdwr, a bole amoniac, a dragon's blood, a chy. mysgweh ychydig spirit of wine gyda hwynt. Profwyd hwn i fod yn effeithiol.

\section{Rhag dolur meun C'ég.}

Cymerwch bowdwr gwin, ac alm, a bole amoniac, a mêl, yr un faint o bob un, a rhwbiwch y gums neu gig eich danedd â hwynt, ac na lyncwch $y$ poer, ond gadewch iddo redeg allan.

\section{Rhag y Dropsy.}

Cymerwch y rhisg melyn oddiar goeden ysgaw, a bay leaves, a chribau Sant Ffraid, a chanomile, a moron gwylltion, a celandine, a rhisg ysgaw Mai, a'u cymeryd yr un modd a'r lleill; a gwraidd eringo, neu fôr-gelyn, a juniper-berries, a phersly, a paladr y wal, a dail llyriain, a goraiad dail tafollydain, 
a smallage. Pob un sydd yn meddwl fod y Dropsy yn dechreu arnynt, y mae yn werth eu trafferth i ferwi yn fanwl y dail hyn ynghyd mewn llestr cauedig, am hir amser, ar dân araf, ac wrth eu potelu rhoddwch ddwy wns o fêl at bob peint, ac yfwch o hono yn fynych; ond yn enwedig yn y boreu ar eich cythlwng.

\section{I stoppio y Terms, sef y Misgluyf, fel y canlyn.}

Cymerwch ddail beets cochion, a dail mieri, $a$ dail cwmphrey, ac o'r dail a elwir haner lleuad. neu Moon wort, ac o lysiau'r gwaedlif, ac o'n dansi gwyllt, ac o'r rosus cochion, at yr un faint a bob un, a'u berwi mewn gwin coch, os gellwch e: gael, (y rhai na, allant gael gwin coch, berwch mewn dwfr), a rhoddwch haner pwys o siwgr gwyr at bob chwart, ac yfwch yn fynych o hono wrth angen, a hyn, gyda chymorth Duw, a'ch gwna yu gwbl iach.

\section{$\boldsymbol{U}$ arall i't un dyben.}

Cesglwch y dansi gwyllt yn leision, os gell wch eu cael, a gwisg wch o dan wadnau eich traed, nesaf $i$ 'ch croen yn eich esgidiau, a hyn sydd yn anffaeledig.

Mae y cyngor hwn yn fwy ei werth na phris y llyfr i gyd.

\section{Un arall i'r un dyben.}

Cymerwch rosus cochion a chwedi eu cynhauafu, powdrwch, a rhoddwch trwy ogr; a chymerwch o hono ddwy lonaid llwy dea ar lasiaid o. win coch, yr hyn sydd anffaeledig. 


\section{Cyngor i Blant wedi tori ea Lleingig}

Cymerwch Solomon seal, a chribau Sant Ffraid, a dail mieri, a'r dansi gwyllt; cesglwch yr un faint a bob un, a berwch mewn dwfr, a rhoddwch ynddo siwgr gwyn, ac yfwch yn helaeth o hono yn dwymn, a rhoddwch y dail yn blastr o'r tu allan am naw niwrnod.

\section{Thag y Canger.}

Cymerwch agrimoni, sef llysiau y Dryw, ac yfwch y dail yu lle tea, ac amserau eraill hefyd, a shoddwch yn blastr ar y dolur; a'i newid bob yu ail diwrnod, hyd nes y byddoch iach.

\section{I gryfhau y Golwg.}

Cymerwch y dorfagl, ac yfwch yn lle tea yn fynych, a rhoddwch y sug yu y llygaid, a hyn a wna y tro i'r hen bobl yn lle prynu gwydrau.

\section{Rhag ysgafnder mewn Pen.}

Cymerwch lonaid llaw o fôm, ac yr un faint o lysiau'r ychain, ac o gribau Sant Ffraid, ac o lysiau'r fam, ac o rosus cochion, a'u berwi oll ynghyd, a'u hyfed fel diod gyffredin yn dwymn, ac yn fynych.

\section{Rhag y Colic.}

Cymerwch lysiau'r angel, a chamomile, a gwraidd rhedyn y dwfr, a haner yr un faint o riw y gerddi, a berwch mewn gwin gwyn, neu frandy, ac yfwch 


\section{4}

lasiaid o hono yn y ffit, neu berwch mewn dwfr, a chymerwch mewn gwin gwyn neu frandy, ac ail gymerwch os na thyr y tro cyntaf.

\section{Fihag dolur mewn Pen.}

Cymerwch rosmari, a churwch mewn morter, a rhoddwch mewn teapot, neu lestr cauedig arall, yn ochr y tân, am wythnos, a gwin gwyn arnynt, ac wedi i chwi wneuthur byny, rholdwch ferw arnynt, straeniwch a phottelwch, a chymerwch o hono lonaid llwy fwrdd nôs a bore; ond llai i blant, fel ag y byddo eu hoed. Ond cofiwch beidio a gadael iddo wastio yn agos i'r tân.

\section{Rhag i blant bisn yn eu guelyau.}

Rhoddwch iddynt lonaid llwy fwrdd o sug llysiau'r gwaedlif, gwedi ei felysu â siwgr gwyn, bob nôs hyd nes y peidiont.

\section{Rhag y Llyngyr.}

Cymerwch gribau St. Ffraid, a gwraidd rhedyn, a wermod lwyd, a chwerwlys yr eithin, a bayleaves, lly siau'r fam, a surans y $\mathbf{c} \hat{w} n$, teim gwyllt, a theim y gerddi, a'r ferfain, a berwch at yr un faint o bob un, a haner yr un faint o riw'r gerddi mewn $\mathrm{dwfr}$, a'i wneud yn gryf, ac yfwch o hono haner peint bob bore; os i blant, gwnewch yn wanach, a rhoddwch lai o hono.

\section{Rhag Crygni.}

Cymerwch flodau carn yr ebol, neu eu gwraidd, 
ac isop, a licrice powder, o siop y Druggist, a mwstard gwyllt y cloddiau, a hâd danadl poethion, a'u cyd-ferwi, at yr un faint o bob un, ac yn niwedd $y$ berwi, rhoddwch bedair wns o fel, a dwy wns o siwgr candi at bob chwart; a phottelwch, ac yna yfwch lonaid cwpan dea o hono hwyr a bore; a byddwch iach.

Un arall rhag givichian a chrygni meun guddf truy ddiffyg anadl.

Cymerwch hâd ffenigl wedi eu powdro a'u cymysgu mewn mêl, a chymerwch o bono lonaid llwy dea dair gwaith yn y dydd.

\section{Diod i iachau briwiau.}

Cymerwch o eiddew y ddaeas, sefllysiau'r esgym, ac o gribau St. Ffraid, a gwraidd dail tafol cochion, a'r bengaled, a'r gantwll, a berwch yu nghyd mewn diod fain, a rhoddwch furum ato, ac yfwch o hono haner peint yn dwymn, nôs a bore, ac iach a fyddwch.

\section{Rhag y Clwyf Melyn.}

Cymerwch surans y cwn, a llysiau y! ychain, a gwraidd banadl, a chelandine, a gwraidd ysgall bendigaid, a gwraidd ffenigl, hops, a horound, llonaid llaw o bob un, a berwch mewn pedwar chwart o ddwfr, nes yr elo yn dri chwart, a rhoddwch ynddo bwys o driagl; ac yfwch o hono haner peint yn dwymn yn gyntaf peth yn y bore, ac yn ail yn y nôs. 
Un arall at yr un achos.

Cymerwch y wermod lwyd, y wermod wen, a Salomon seal, a'r valerian, a surens y gôg, a'r pren melyn, a chribau St. Fraid; cesglwch at yr un faint - bob un, a naddwch y pren melyn yn ysglodion mâu, yr un faint ag o'r lleill; a'u rhoddi yngwlych dros nôs mew'n pedwar chwart o hen gwrw ; ac yn y bore berwch ar dân araf, hyd nes iddo leihau un chwart; ac yn niwedd y berwi, rhoddwch ynddo haner owns o saffrwn, berwch drachefn am bum' munud, a thynwch i lawr wedi ei gau yn glos, a phan yroero, ystraeniwch a phottelwch, a chymerwch o hono lonaid llwy yn dwymu.

\section{Rhay Piso Gracd.}

Cymerwch lysiau'r dryw, a dail llwyn hidl, teim y gerddi, teim gwyllt, a dail rhosus cochion; llonaid llaw o bob un, a berwch hwynt mewn peint o ddwfr, nes iddo leihau un peint, a rhoddwch ynddo haner pwys o siwgr gwyn, ac yfivch - hono haner peint hwyr a bore yn dwymu.

\section{Rhag y Ddannodd.}

Cymerwch lysiau'r angel, a bôm, a dail mieri, yr un faint o bob un, yn leision, a gwasgwch eu sug, a rhoddwch yn nhwll y daint, yua iach a fydd.

\section{Un arall ir un achos.}

Cesglwch deim gwyllt, a theim y gerddi, gwasgwch eu sug, a hyn a i hesmwythâ; ond y cyngor goreu yw tynu y dant, os bydd yn llygru, rhag iddo lygru un arall a fyddo yn nesaf ato. 
1 attal Cyfog, ac i gryfhau'r Ystumog.

Cymerwch ddail llyriaid, dail llwyn hidl, mintys, bôm, cribau St. Ffraid, dail rhosus cochion, dail derw, brenhines y weirglodd, a rhosmari; cesglwch lonaid llaw o bob un, a berwch ynghyd mewn pedwar chwart o ddwfr, nes elo yn dri chwart, ac yfwch o hono lonaid cwpan dea dair gwaith yn y dydd.

\section{I wellhau $y^{r}$ Ysplin.}

Cymerwch wraidd march alarch, a dail chamomile, a gwraidd eringo, sef celyn y môr, a gwraidd rhedyn y dwfr; Cymerwch haner pwys o'r tri cyntaf, a chwarter pwys o wraidd rhedyn, mewn pedwar chwart o ddwfr, a berwch nes iddo leihau yr haner; ac yn niwedd $y$ berw rhoddwch ynddo beint o wîn gwyn, a phwys o siwgr coch, ac yfwch o hono lonaid cwpan dea, nôs a bore, yn dwymn.

\section{I ostwng Chwydd yn y Corph.}

Cymerwch ferw y dwfr, a lercitus, a danadl, a camomile, a dail llyriaid, y wermod, yr un faint o bob un, ond y danadl a'r wermod, a haner yr un faint o's ddau olaf; berwch mewn diod fain, a rhoddwch ynddo fêl, ac yfwch o hono yn dwymn wrth eich angen" a'ch deall:

\section{I ostwng Chwydd allanol.}

Cymerwch ddail ysgaw, a dail gwywydd, a llysiau y feddyges, llonaid llaw o bob un, a berwch mewn brecci melus, a thewychwch ef â blawd 
brâg, a blawd rhyg, yr un faint o bob un, a thodd wch fêl ynddo, a gwnewch yn ystwyth $\hat{a}$ saim gwेydd, oil, neu floneg, a rhoddwch yn blastr ar y lle.

\section{Diod rhag, y Scurvy.}

Cymerwch ferw'r dwfr, a juniper, a dail scurvy, a gwraidd dail tafol cochion, a'u berwi ynghyd, yr un fänt o bob un, nes y byddont yn ddiod gref iawn, a gwnewch yn lled siarp â cream of tartar, ac yfwch o hono jn helaeth nôs a bore.

Cyngor $i$ Ferched pan fyddo eu Terms wedi stopio.

Cymerwch lysiau'r dryw, a llysiau'r esgyrn, a dail Alexander, a bôm, a chribau St. Ffraid a bayleaves a moron gwylltion, a berw'r $d w f$, a gwraidd eringo, sef celyn y môr, a horound, a hâd dail poethion, a juniper, a llysiau Evan, y ferfain, a palat y wal, a lercytus, a sage, a dail gwywydd, a wermod, a rhiw y gerddi, a phenniw; cesglwch lonaid llaw o bob un ond y rhiw a'r phenniw, haner llond llaw o'r thai hyny; cewch y juniper yn siop y Druggist, rhoddwch bedair owns o honynt gyda'r dail a enwyd, a berwch mewn galwyn at haner o ddwfru ar dân araf, a chauad clos ar ei wyneb hyd nes iddo leihau i un galwyn, a rhoddwch, wedi y berwoch, ynddo beint o fêl, ac yfwcls o hono haner peint nôs a bore yn dwymn.

Rhag Dolur y Gareg a phoen yn y cefn.

Cymerwch bey leaves, a blodau danad!, a dail llyriaid, a racamwcci, a camomile, a moron gwyllt- 
ion, a dail cherries, a berw'r dwfr, ac ychydig hẩ mwstard, wedi eu sigo, a chlust y llygoden, a hâd danadl poethion, a phalat y wal, a rubob, a gwraidd dail tafol, a surens y gôg, a smallage; cesglwch yr un faint o'r dail byn oll, a rhoddwch ynddo ychydig hâd llîn, a rhoddwch ynddo dwysgen o fêl, ac yfich yn fynych o hono. Da fyddai i bawb sydd a'r grafel arnynt yfed o'r ddiod hon.

\section{Cyngor i Wragedd rhag Misgario.}

Cymerwch sage, a dansi giwyllt, yr un faint 0 bob un, a chnociwch a gwasgwch allan eu sug a chymerwch lonaid llwy fwrdd o hono yn lled fynych.

\section{Rhag Dolur mewn Bol.}

Cymerwch gamomile, a dansi gwyllt; a moron gwylition, a berwch ynghyd, a chymerwch ddracht. helaeth, o houo yn boeth, ac yn fynych.

\section{Rhag y Polsi.}

Cymerwch gribau Sant Ffraid, a bey leaves, a sage, a dail briallu, cesglwch yr un faint o bob un, ar ôl ell cynhauafa a'u powdro, cymysgwch en powdr yn dew mewn mêl, a chymerwch o hono lonaid llwy dea ddwy waith yn y dydd.

Un arall at yr un achos yn allanol.

Cymerwch rosmari, a sage, a lavender, a bay leaves, a smallage, a camomile, a rhiw'r gerddi, a 
mintys, a wermod, a chribau Sant Ffraid, a lercitus, a'r gantwll; cesglwch lonaid llaw o bob un, a berwch mewn dwfr, a golchwch yr aelodau clwyfus â'r dwfr mor boeth ag $y$ gellir ei ddyoddef, a gorwedd mewn plangcedi ar ei ôl bob nôs.

\section{Rhag y Peils.}

Cymerwch ddail mieri, a dail cwmphri, a dail triagl y moch, (termentil) a dail poethion, a palat y wal, cesglwch lonaid llaw o bob un, a berwch mewn pedwar chwart o ddwfr ar dân araf, ac yfwch haner peint o hono hwyr a bore, a byddwch iach.

\section{Eli i iachau y Peils.}

Cymerwch ddail llygaid y dydd, a llygaid Ebrill neu ddail peils, nid rhaid $i$ chwi ond codi eu gwraidd, cewch weled dull y peils arnynt, ac yna cymerwch eu gwraidd, a'r un faint o lygaid $y$ dydd, a churwch ynghyd, a berwch mewn bloneg hyd nes b'ont yn crimpio, ac jna straeniwch ac irwch rhag y peils âg ef.

\section{Rhag y Melancoli.}

Cymerwch fôm (balm,) a llysiau'r fam a llysiau'r ychen, at yr un faint o bob un, a berwch yn nghyd mewn dwfr a gwin gwyn, ac yfwch fel diod gyfiredin; neu bowdrwch y dail a chymysgwch mewn mêl, a chymerwch ar lwy dê amrywiol weithian yn y 
dydd; nou cymerwch un dram o'u powdr ar wîn gwyn ddwy waith yn y dydd, a dyma'r ffordd oreu yn ol fy marn i.

\section{I gryfhau y Cöf.}

Cymerwch y dorfagl, a gwraidd yr ysgall bendigaid, a Juniperberries, a lili'r dwfr, a chasglwch yn nghyd, yr un faint o bob un, a berwch ar dâu araf, a gwnewch hwynt fel diod fain, ac yfwch wrth eich syched.

\section{I ychwanegu Llaeth.}

Cymerwch lysiau'r ychen, a ffenigl a dail y rhoccos, yr un faint o bob un, a berwch mewn llefrith; ac yfwch fel y b'o yn angenrheidiol.

\section{Rhag ffitiau, neu wynt y fam.}

Cymerwch arac, sef llysiau'r fam wyllt, neu droed yr wydd, a llysiau'r fam ddof o bob rhyw, a moron gwylltion a phalat y wal, cesglwch at yr un faint o honynt oll, rhoddwch ddwfr berwedig arnynt mewn llestr cauedig, a chedwch mewn gwres ac yfwch o hono wrth eich angen.

\section{Cyngor $i$ wellhau y Manwynau.}

Cymerwch wraidd March-alarch, a churwch yn nghyd $\hat{a}$ halen ac ychydig finegr, a rhoddwch ar $y$ dolur cyṇ iddo dori, ac fèi gwellhâ yn fuan. 
Un arall.

Cymerwch daail bysedd cochion, a churwch, a a berwch mewn dwfr, a gwnewch bowltis, gydâ blawd ceirch, a daliwch wrtho hyd nes y toro, ac ar ol iddo dori efe a'i sugna, ac a'i sycha, ac a"i croenia yn gwbl iach.

\section{Un arall.}

Cymerwch Iysiau Evan, a gwraidd eringo, neu gelyn y môr, a'u curo yn nghyd â bloneg, a'i newid unwaith yn y dydd.

\section{Rhag yr Igian.}

Cymerwch wraidd neu hâd fenigl, a dail mintys, a'u berwi ynghyd mewn.dwfr, ac yfed o hono yn dwymn.

\section{I iachau y Pox.}

Cymerwch wraidd celyn y môr, a sage gwylltion, a hops; o gelyn y môr, tri pwys, ac un o sage, a chwarter pwys o hops, a'u berwi mewn pum' chwart o ddwfr, nes eu lleihau i bedwar chwart, a'u hidlo, a rhoddi ynddo bwys o driagl, a'i weithio $\hat{a}$ burum, a'i yfed fel diod gyffredin, ond rhaid da ato am hîr amser.

Cyngor i rai sydd a'u gwynt yn drewi.

Cymerwch fintys, a rosmari, a berwch ynghyd, a golchwch eich genau âg ef bob bore. 
Un arall.

Cymerwch hâd smallage, wedi eu powdro, a'us cyneryd mewn mêl, neu eu berwi newn dwfr, a'u hyfed yn fynych.

Un arall $i$ wellhau guynt a f'o'n drewi truy'r Canger.

Cymerwch sug surans y gôg, a mêl, a golchwch eich genau â hwynt yn fynych.

\section{Cyngor i wellhau y Frech goch.}

Cymerwch wraidd triagl y môch, (tormentile) a berwch ddau bwys o honynt, wedi eu glanhau mewn tri pheint o ddwfr, nes elo yn chwart, a rhoddwch ynddo haner pwys o driagl, ac yfwch o hono yn gynes lonaid cwppan dea, dair gwaith yn y dydd, a hyn a'i teifl allan yu brysur ac a iachấ' galon.

\section{Rhag y Frech Wen.}

Cymerwch haner owns o flodau gold mair, a haner owus o saphrwm, a rhoddwch arnynt ddau chwart o ddwfr berwedig mewn llestr cauedig, a'i gadw ynghwr y tân, a rhoddwch ynddo haner pwys o fêl, ac os i blentyn y bydd, rhoddwch iddo lonaid Hwy fwrdd yn lled fynych; ac os i un yn ei oed a'i nerth, geill ei gymeryd yn helaethach.

\section{Cyngor $i$ rai trwm eu clywed.}

Cymerwch sug llysiau'r angel, a thywalltwcls Wrechydig o hono i'ch clustiau bob nôs, a rhoddi Hswlân yoddynt, a chadw cynesrwydd. 
$U_{n}$ arall.

Cymerwch o sug savory, ac oil of roses, a'u cymysgu ynghyd, yr un faint o bob un, a'u rhoddi yn dwymn yn y clustiau bob nôs, a chadw cynesrwydd; a hyn a dỳr y sŵn yn y pen.

\section{$\boldsymbol{U}$ a a all "̈r un dybes.}

Cymerwch o sug ysgaw Mai, a sug celyu y môr, yr un faint o bob un, a dyferwch i'r clustiau, a chadw cyuesrwydd.

\section{I iachau y Bysen mewn Llygad.}

Cymerwch o sug llysiau'r angel, ac ysgaw Mai, a calendine, a'r dorfagl, yr un faint o bob un, a'u cymysgu ynghyd âg ychydig fêl, ac ychydig laeth bron, a'i ddyferu i'r llygaid yn fynych.

I wellau y Polsi sydd yn gwneud i'r dwylaw grynu.

Cymerwch flodau ysgaw Mai, a'u distilio yu mis Mai, a golchi'r dwylaw âg ef nos a bore, hyn a'u gwellâ, ac a gryfhâ'r gewynau.

\section{Rhag y Gout.}

Cymerwch ddail llygaid y dydd, a chribau St. Ffraid, a bôm, a chrafangc yr arth, a berwch oll mewn dwfr, a golchwch $y$ chwydd $\hat{a}$ hwynt $y n$ fynych. Neu cymerwch ddail liggaid y dydd, a berwch mewn bloneg nes y byddo'r dail wedi crimpio, ac yna ystraeniwch trwy liain, ac irwcl \$ Gout â'r enaint. 
1 chwalu gwynt o'r Ystumog neu'r cylla.

Cymerwch rosemari wedi eu powdro, a'u rhoddi trwy ogr, a chymerwch haner dram o honynt nos a bore, ar win gwyn.

\section{Eli da i lanhau Briwiau.}

Cymerwch haner pwys o sug smallage, ac o fêl, bedair wns a haner, a blawd gwenith, wns a haner, a berwch hwy ynghyd hyd nes y tewychont, a chedwch hwynt $i$ lanhau hen friwiau dyfrllyd a drewedig.

Urs arall i'r un dyben, ond yn fwy effeithiol i fwyta cig marw.

Cymerwch bedair wns o sug smallage, a'r un faint o sug safander, ac un wns o verdigrise, ac o fêl, bedair wns, ac o winegr, dwy wns, a'u berwi yngbyd ar dân araf hyd.nes y tewychont. Detnyddiwch yr un modd a'r llall.

Nid yw yu berthynol i un math o friwiau ond in rhai sydd a chig marw ynddynt.

Enaint $i$ wellâu cymalau ac aelodau wedi vhwymo gar oerfel.

Cymerwch o beyleaves, haner pwys, yn leision, ac o ddail cabbage, chwarter pwys, yn leision, a'u berwi mewn pum pwys o saim traed defaid, a dau bwys o wêr eidion, a berwch y dail ynghyd yn y saim a'r gwer, hyd nes y crimpo y dail ynddo, yna straeniwch, a chedwch yn barhaus i iro'r gewynau a'r aelodau clwyfus, pan fo'nt yu pallu gan oerfel. 
Enaint i'r Nerves, ac $i$ wellhau holl ffaeledd yr aelodau trwy oerfel.

Cymerwch sage, a rhosemari, a lavender, a beyleaves, a chamomile, a rhiw'r gerddi, a wermod, llonaid llaw o bob un o'r dail hyn, ac o fintys, ac o gribau St. Ffraid, y gantwll, a phersly, haner llonaid llaw o'r rhai hyn; saim traed defaid, pum pwys, gwêr defaid, dau bwys, oil spike, haner wns, a'r dail wedi eu curo a'u berwi ynghyd a'r saim, yr oil, a'r gwer, wedi iddo ferwi digon, straeniwch trwy liain, a chedwch fel peth ofawr werth i eneinio'r aelodau pan y byddont glwyfedig a diffygiol. Pa beth bynag yw, os bydd wedi magu trwy oerfel, neu anystwythder yn y cymalau, neu'r aelodau yn diffrwytho gan $y$ polsi, neu ryw beth arall o'r cyfryir; os bydd y Colic, neu wynt yn y bol, gellivech yn llwyddianus wneud defnydd o'r enaint - flaen y tân, a chadw cynhesrwydd. Dyma gyngor o fawr werth.

Todd $i$ wneuthur Plaster $i$ dori y Colic, as $i$ esmwytliau poen yn mhob rhan o'r corph.

Cymerwch y berries, neu y grawn sydd yn tyfu ar y bayleaves, ac os na ellwch eu cael, cymerwch y dail wedi eu powdro, ddwy wns, ac o venus turpentine, dwy wns a haner, o Frankin-sense a mastic, haner wns, ac o fêl, bedair wns, a gwnewch hwynt yn blaster, trwy eu cymysgu a'u berwi ynghyd, a'u taenu ar ledr, a'u rhoddi ar y lle clwyfus, pa un bynag ai ar y bol neu ar yr aelodau. $Y$ mae liwn wedi bod o fawr leshad amrywiol droion. 
Plaster da z roddi ar Esgyrn Briwedig, ac $i$ lanhaw hến friviau.

Cymerwch gribau St. Ffraid, yn leision, a llysiau y dryw, sage, lercitus, llysiau y givaedlif, a chwmphri, a'u curo a'u berwi mewn peint o win gwyn; pan ferwont, streiniwch, a thefiwch y dail ymaith, a chymerwch o frankinsense a mastick, wedi eu powdro, o bob un, chwarter wns, a chwyr gwyn a thurpentine, o bob un bedair wns, ac ystor, ddwy wns, a berwch yr holl bethau uchod yn y gwin, yn yr hyn $y$ berwyd y dail, a gwnewch yn blastr a rhoddwch ar ledr ar y lle briwedig; gellwch wneuthur o hono mor lleied a fynoch, ond cymeryd llai. o'r definddiau.

Y ffordd $i$ uneud Eli da at bob math o Friwiau.

Cymerwch haner pwys o floneg wedi ei doddi, ac o blwm coch, ac o blwm gwyn, (read and white lead), wns a haner o bob un, ac o gîyr melyn, wns a haner, ac o ystor du, un wns, o venus turpentine, ddwy wns; berwch hwynt am dri chwarter awr, a rhoridwch y vellus ynddo, agos ar ddiwedd y berwi, a'i dynu i lawr, gan ei droi hyd nes oero.

Plastr da i roddi ar Esgyrn briwedig i feddalhau ac i ostwng chwydd, ac i dymheru rhyw ran ar y corph a fyddo wedi ei niweidio trwy oerfel; gellwch ei gael yn Siop y Druggist, dan yr enw oxycroceum plaister.

\section{Rhag Ysgyrfi yn y Dannedd.}

Cymerwch sage tê, a rhoddwch ynddo ychydig al. lum i'w doddi, a chymerwch gadach lliain, a golch. 


\section{8}

wch gig eich daneddág ef yn dda ac yn fynych; ac os ewyllysiwch wneuthur eich dannedd yn wynion, cymerwch allum wedi ei losgi a'i bowdro, un wns, a chwe Ilwyaid o s!g calendine, cymysgwch ynglyd, a rbwbiwch eich damedd âg ef.

\section{Cyngor rhag y Dufr poeth.}

Cymerwch ychydig salts of tartar, ac yr un faint o'r magnesia, a'u cymysgu mewn ychydig ddwfr, a chymeryd llonaid llwy fwrdd o hono yn lled fynych; hyn a wellha y Dwfr poeth.

\section{Cyngor $i$ rai sydd dueddol i fod yn rhwym.}

Cymerwch cream of tartar wedi ei gymysgu mewn mêl, a'i gymeryd yn fynych yn y bore: a hyn a geidw y corph yn dymherus.

I ostwng chwydd yn mronau merched, pan y byddo o herwydd gormod o laeth.

Cymerwch gîyr melyn, ddwy wns, ac o linseed oil, neu olew had llin, haner pwys, a thoddwch ynghyd, a gwlychwch liain glân ynddo, at yr un faint a'r lle dolurus, pan ag y byddo yn dwymn, a rhoddwch ar $y$ fron, neu ar $y$ ddwy, os bydd yn angenrheidiol; a gwnewch hyn nes darfyddo y boen, a gostwing y chwydd.

Cygarwyddyd $i$ Fammaeth sydd ry brin o laeth $i$ iv phlentyn.

Deallwch gymaint a hyn, os bydd y Fammaeth yn gystuddiol, ac yn cario meddyliau trymion, y 
mae hyn yn ei gwneuthur yn deneuach; nis gall fod ganddi lawer o laeth, a'r hyn sydd ganddi, ni all fod yn llaeth iach, ac y mae bwydydd a diodydd sâl yn lleihau y llaeth; am hyny dylid ymorthod $\hat{a}$ phob bwydydd drwg ac afiach, a buyta y bwydydd goreu os gellir eu cael, a'u diod addylai fod o lefrith da; yn mha un y dylai fod yngwlych had ffenigl dros nos; a barley water, neu ddwfr haidd sydd dda hefyd i'r un dyben. Neu gymeryd barley water, a rhoddi o ddail ffenigl yn leision ynddu, a'u berwi, a'i felysu $\hat{a}$ siwgr, a'i yfed fel diod gyffrediu; ac yn allunol, cymerwch frenigl a smallage, yr un faint o bob un, a's berwi mewn dwfr, a golchi y frest âg ef nor boethag y gellir ei ddioddef; neu curwch ddail mintys, a rhoddwch yn bowltis poeth ar y fion; nea cymerwch phenigl a phersly, lonaid llaw o bob un, yn leision, a'u curo ynghyd, a'u chwysu, a'u rhoddi yn bosltis cynes ar y fron, a hyn a ychwanega $y$ llaeth.

\section{Cyngor $i$ wellhau y Paps, nea ben y grono}

Gwnewch bowltis o thawd $\mathbb{T a}$, a salad oil, a rhoddwch yn gynes ar y dolur, a newidisych ef un waith yn y dydd, ac os bydd pen y fron yn chwydd caled, cymerwch lonaid llaw o ryw'r gerddi, a'r un faint o horownd, ac o fintys, a churwch y dail ynghyd meirn mêl, a rhoddwch yn bowltis ar y lle, hyd nes y byddo yn iach.

I dori y Colic ar ferched, pan y byddo eas hamser yn agoshau i eni plarit.

Y mae y boen hon, ar mai anserau, mor annioddefol a'r boen wrth esgor, ac y mae'r fydwraig 
anneallus yn anl yn cymeryd y naill yn lle y llalis ac ar rai gweithiau y maent yn digwydd yn yr un munudau, yr hyn sydd yo gwneuthur en poen yn liawer gwaeth, ond i brysuro at y cyngor; cymerwch ddwy wns o oil sweet almonds, ac un wns o sinamon water, a phedwar diferyn o spirit sunsur, ac i'r ferch ei yfed i gyd pan yn y fit o'r Colic.

\section{Rhag dolur mewn boliau plant bychain.}

Os bydd o herwydd y llyngyr, cymerwch gamomile, a rhiw'r gerddi, ac ychydig o fustl eidion, a'u curo a'u berwi yn nghyd, a'u gwneuthur yn bowltis i'w rhoddi wrth y bol; os bydd ar ryw achos arall, rhoddwch iddynt un dram o oil sweet almonds, a siwgr candi. Gellwch hefyd yn llwyddianus roddi iddynt haner dram o had anis wedi eu powdro, a'u cymeryd gydâ rhyw beth cyfaddas.

I gryfhau plant bychain, pan $y$ 'byddont weiniaid newydd eni.

Cymered y fudwraig, neu ryw un arall, y plentyr mewn gwlanen gynes at y tân, a chymered ychydig wîn coch yn ei genau, a rhoddi o’i genau ei hum jn ngenau y plentyn; gwnewch hyn yn fynych, a a rhoddwch liain wedi ei wlychu mewn gwîn coch cynes ar fol a brest y plentyu.

Rhag ffitian Confulsiwn ar blant bychain newydd eu geni.

Deallwch nas gall plentyn newydd ei eni gymeryd $\operatorname{dim}$ yn dufewnol, ond yn allanol: cymerwch 
flodau camomile, a gwraidd lili y dwfr, a berwch ynghyd mewn dwfr, a golchwch y plentynâg ef yox dwymn, yn fwyaf neillduol asgwrn y cefn, ac ar ol ei olchi ef, eneiniwch drachefn $\hat{a}$ saim gồydd, a rhwymwch ef mewn gwlaneni i'w gadw yn gynes: ac os na wna y pethau a enwyd y tro, cymerwel oil of worms, neu olew pryfed genwair, ac oil of lilies, ac oil of foxes, ac oil of turpentine; y mae rhai neu'r cwbl o'r oelion hyn yn dda i eneinio asgwrn y cefn o flaen ychydig wres, a'i rwymo of mewn gwlanen. Gellwch ddefnyddio y cyngorion hyn at blant a fyddo'n hynach, yn gystal ag i rai fyddant newydd eu geni, os byddant yn dueddol i'r ffitiau confulsiwn. Deallwch hefyd fod yr oelion hyn; a phob peth arall a enwyd yn y llyfr hwn ond y dail, i'w cael yn siop y Druggist.

\section{Ychydig gyfarwyddyd i gymergd Purge.}

Deallwch hyn, os bydd eich cyrph yn bur rwym, ac yn ffaelu ei ryddhau gyda rhyw beth yn raddol, ac yn gorfod cymeryd purge cryf; byddwch yn sicr o ddefnyddio glister o'i flaen, i agoryd y ffordd, os bydd y ffordd, passage, wedi cau, y mae yn rhy bergglus cymeryd purge cryf, ihag iddo weithio yr afiechyd ryw ffordd arall; a chodi y colic new y confulsiwn, neu beth gwaeth yn ei le, o herwydd mawr ydyw y niwed $y$ mae purge cryf yn ei wneuthur ar gyrph dynion; byddwch ddwy awr ar ol cymeryd purge, cyn yfed dim ar ei ol; gadewch i'r cylla, sef yr ystumog, fod yn wâg pan ei cymeroch ef, rhag id̛do gymýsgu â'r ymborth, a thrwy hyny golli ei nerth, ac anmhuro y gwaed, na chymerwch ddim a f'o'n felus ar ei ol:y mae 


\section{2}

purge yn gwanhau y galon a'r natur, am hyny cymerwch ryw beth cyfaddas gydâ'ch purge a fyddo yn cryfhau y galon a'r cylla: os bydd yr afiechyd yn y pen, neu yraelodau gwell ydyw fod eich Purge wedi ei wneyd yn galed, megis Pills, neu ryw beth cyffelyb, os yn y bol, gwell yw ei yfed.

\section{Dull Arac wild, neu Lysiau'r Fam wyllt.}

Y mae y rhain yn ddail mân, ac agos yn grynion, ond ychydig yn fain at y blaen, heb ddim toriadau, na bylchau ynddynt, yn tyfu ar wreiddyn eiddil; a r cangenau yu ymdaenu ar $y$ ddaear, ac y mae yr holl ddeilen yn arogli fel pysgodyn drewedig, neu rym beth 'gwaeth.

Y maent yn tyfu yu gyffredin ar y tomenau, yn blodeuo yn Mehefin a Gorphenaf; a'u rhinweddl fel y canlyn, sydd $\mathrm{i}$ iachau merched pan y byddont bron wedi gwallgofi gan ffitiau y Fam wrth eu harogli; ond yn enwedig wrth en cymeryd yn dufewnol. Nid oes yn,tyfu dan yr baul na'r lleuad mo'w gwell rhag y dolur hwnw, sef gwynt y fam. Myfi a helaethwn ar y ddeilen hon pe byddai genyt ddoniau. Y mae hi i'w chael yn gyffredin ar $y$ tomenau: y mae.hyn yn galw am ein diolchgarwch, trwy ddangos i ni fod gwaith Duw yn rhâd ac yn helaeth i ddynolryw. Myfi a gymellaf y ddeilen hon i chwi megis un o'r perlau mwyaf ei goerth i fod yn anffaeledig feddy giniaeth i holl ddoluriau $y$ groth, y fath a iachâ yu rhwydd, yu sicr, ac yir brysur holl ffaeledd y groth; $y$ maent yn oeri'n groth pan y byddo yn rhy boeth. gadewch i mi, a mi a ddywedaf $i$ chwi y gwir, mai poethder y groth yw. un achos, ac achos mawr, fod gwragedd yn cael 


\section{3}

esgorfa mor galed ar enedigaeth plant: ac y maent hefyd yn rhinweddol $i$ wneuthur $y r$ anmhlantadwy yn ffrwythlawn, trwy eu bod yu cryfhau ac yn glanhau y groth: ac yn mhellach, y maent yn dyfod a'r cyrsiau misol i lawr ac $y$ byddont wedi ffaelu, ac yn eu hattal, os byddant yn dyfod yn rhy helaeth. Nis gallwch ddeisyf dim daioni i'r groth, nas guna y ddeilen hon yu gyflawn. Am hyny, os ydych yn caru plant, ac os ydych yn caruiechyd, ac os ydych yn caru esmwythdra, cadwch y dail hyn yn eich tai trwy y flwyddyn.

Y FFORDD I'W DERNYDDro. Os bydd y dail yn leision, curwch a gwasgwch eu sug, a chadwch yu eich tai trwy y flwyddyn; cewch gyfarwyddyd jn niwedd y Llyfr i gadw y sug yn ddilwgr am flwyddyn, ac os bydd y dail wedi eu cynhauafa, berwch hwynt mewn dwfr neu win, a rhoddwch ynddo yn helaeth o siwgr coch, ac yfwch yn fynych o hono wrth eich angen. Byaded i'r cyfoethogion ei gadw i'iv gyfranu i'r tylodion. 


\section{BRITISH IIERAL}

\section{NEU}

\section{LYSIEULYTR BRYTANAIDD}

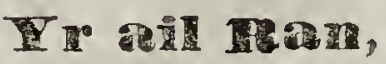

\section{YN CYNNWYS 50 O LYSIAU MEDDYG- INIAETHOL,}

A'n hamrywiol rinweddan i symud ac i wellhau yr amrywiol ddolurian a'r afiechyd ag y mae dynolryw yn ddarostyngedig iddynt.

Inghyd a chyfarwyddyd i gasglu, i sychu, ac i gadw pob rhan o'r llysiau, a'r fordd i dynu eu sug, i'w puro, ac i'w cadw yn ddilwgr, a'r frordd $i$ wneuthur suryp o konynt.

Hefyd cyfarwyddyd $i$ adnabod natur y llysian, y rhai poethion a'r ruai oerion ar 'el penau eu hunain, a'r ffordd ' ${ }^{\prime} w$ defnyddio fel ac y byddont yn gyfaddas feddyginiaeth i'r clwyf, pa un bynag ai yn dufewnol, ai yn allanol y byddont.

Cyfarwyddidau i gasglu, $i$ sychu ac $i$ gadw y dail a'r sug yn ddilwgr an floyddyn.

Am y dail, cesglwch y rhai sy'n leision ac yn lawn o sug, a phigwch yn fanwl y gwywedig a'r llygredig i'w taflu allan fel pethau o ddim gwerth, onid ê fe lygra y lleill oddiwrthynt; a chasglwch $\mathrm{y}$ dail cyn iddynt flodeuo na hadu, oherwydd fod eu nerth yn myned i fynu i'r topiau; ac os na bydd cyfleusdra yn rhoi i'w tori cyn iddynt ehedeg, cymerwch eu topiau a gwnewch ddefnydd o honynt, a sychivch y dail yn yr haul, a rhoddwch hwynt mewn cadachau o bapur, a gwniwch hwy i fynu, a chedwch yn agos i'r tàn mewn lle sych. Nis gallwn ddywedyd yn gywir am eu parhâd. mewn nerth, oherwydd eu bod yn wahanol i'w glydd; y rhai a heliwch mewn tir sych a geidw yn 


\section{5}

hwy na rhai o dir llaith. Gellwch ddeall paw gollont eu lliw a'u harogl y byddant wedi colli eu nerth hefyd, na wnewch ddefnydd o'r rhai llygredig, rhag idynt lygru eich cyrph fel ag y maent eu hunain yn llygredig.

\section{Am y Blodau.}

Y Blodau yw harddwch y llysiau, ag y maent i'w casglu pan fyddont yn eu prime, a chesglwch hwynt pan fyddo yr haul yn tywynu arnynt, ac us chesglwch hwyrt pan y byddont yn wlybion gan na gwlaw na gwlith; os heliwch hwynt yn wlybion ni chadwant ddim yn hir. Cynhauafwch hwy yn yr haul, a chedwch hwy mewn cydau papyr yn agos i'r tân, fel y dangosais o'r blaen; ac mor hir ag y cadwant eu harogl hwy a gadwant eu nerth.

\section{Am yr Hadau.}

Deallwch hyn, fod holl nerth y llysiau yn yr hâd: sychwch ychydig arnynt yn yr haul, a chedwch hwynt, fel ag y dangosais o'r blaen; ond ni raid i chwi ofalu cymaint am eu cadw yn agos i'r tân, o herwydd eu bod yn gryfach na'r lleill, ac a gadwant lawer o flynyddoedd; ond gwell ydynt y flwyddyn gyntaf.

\section{Am y Gwraidd.}

Dewiswch y rhai anllygredig o honynt, a'r rhai heb gael eu bwyta gan bryfed, a'r rhai sydd yn eu lliw, eu blas, a'u harogl, ac heb fod yn rhy galed nac yn rhy feddal; cesglwch hwynt ar amser sych, a'r rhai sydd yn feddal o honynt sycliwch yn ngwres yr haul, neu ar linyn yn nghongl $y$ simnai; ond am $y$ 


\section{6}

thai caled, gellwch eu sychu ifynu fel y mynoch, ond ofer yw eich gwaith i sychu $\mathbf{y}$ rhai sydd i'w cael trwy y flwyddyn, ond eu cael pan bo eisiau.

\section{Am y Sug.}

Y Sug sydd i'w wasgu allan o'r dail, neu'r blodau, neu'r topiau, neu rhyw ran arall o'r llysiau pan y bydaiont yn ieuangc ac yn dyner, a heliwch $y$ dail pan na byddont wlybion gan na gwlaw na gwlith, onid ê ni bydd y sug o ddim gwerth i'w gadw, curwch $\mathbf{y}$ dail a gwasgwch allan y sug trwy gwd-lliain, a phurwch ef, sef tynu y sothach allan $\alpha$ hono, fel y canlyn, rhoddwch ar y tân, mewn ysgelet, a berwch, a phan godo y ffroth, heliwch i'w datlu ymaith; a deliwch ar y tân nes iddo ddarfợ ffrothio, ac ar ol iddo oeri, potelwch, a rhoddwch an ei dop ddwy fodfedd o sweet oil, yr hwn a geidw yr awyr allan o hono, trwy ei fod yn wastadol yr nofio ar y gwyneb; os caiff gwynt fyned ato ni fydc o ddim gwerth; a phan y byddocli ar fedr gwneuthur defnydd o hono, tywelltwch beth o hono allan a scimiwch yr oil, a rhoddwch ef yn y botel; : hyn a'i ceidw yn berffaith am hir amser.

\section{A $m$ y Suryps.}

$Y$ ffordd i wneuthur Suryp, cymerwch beint o' sug, wedi ei buro yn ol y cyfarwyddyd o'r blaen ac at bob peint rhoddwch bwys o siwgr coch, a berwch hwy ynghyd, a scimiwch y ffroth i'w dafle ymaith, ac wedi iddo ferwi digon, streiniwch trwy lian, a chedwch hyd nes y delo angen am dano; fa geidw an flwyddyn neu ychwaneg, cedwch ef mewn gwydrau neul lestri pridd heb eu corcio; ni raid 


\section{7}

rhwi ond rhoddi papur ar ei wyneb, a'i rwymo âg edef; mae hyn yn llawer mwy rhinweddol na berwi $\mathrm{y}$ dail at y doluriau ag y bont yn berthynol. Os bydd i chwi fanwl chwilio y llyfr bychan hwn, a gwnend yn ol y cyfarwyddyd a osodais i lawr ynddo, gellwch gael budd a lleshad corphorol oddiwrtho pan y byddwyf fi wedi myned $i$ ffordd $y r$ holl ddaear.

Myfi a ddeuaf yn bresenoliddangos i chwi y ffordd oreu i wneuthur defnydd o'r dail, fel y byddont yn fwy cyfaddas at yr amrywiol ddoluriau, pa un bynag ai yn allanol neu dufewnol y bo's dolur; yn gyntaf daliwch sylw ar natur eich dolur, pa un ai poeth ai oer ydyw, a pha un ai trwy ormod o wres neu oerfel y mae y dolur wedi magu, ac os bydd y dolur trwy oerfel, ac wrth natur yn oer, cymerwch y dail sydd wrth natur yn boeth; ac os bydd y dolur trwy wres, ac wrth natur yn boeth, cymerwch y dail sydd wrth natur yn oer; pa un hynag ai yn dufewnol ai yn allanol y byddo'r dolur. Ond i'ch gwneud yn fwy dealladwy yn natur y dail, myfi a roddaf y dail sydd boethion yn eu natur i lawr gyd a'u gilydd, a'r rhai sydd yn oerion yn yr un modd gyd a'u gilydd, fel ag y galloch weled pa rai sydd, a'r thai nad ydynt gyfaddas i'r clwyf. Ond deallwch fod llawer mwy o rai poethion yn eu natur, wedi eu casglu, na'r rhai oerion yn eu natur; oherwydd fod doluriau sydd yn magu trwy oeriel yn fwy aml yn y gwledydd oerion hyn. Myfi a ddangosaf yn bresenol y rhai poethion yn eu natur yn gyntaf, fel y canlyn:-

Llysian y dryw, llysiau yr angel, balm, neu bôm, cribau St. Ffraid, cacamwci, bey leaves, banadl, camomile, carn yr ebol, salendine, neu lym y 


\section{8}

llygad, eringo, sef celyn y môr, y dorfagl, gwraidd rhedyn, ffanigl, llysiau yr esgyrn, horownd, dail y gwinwydd, llysiau y fam, gwallt $\mathrm{y}$ forwyn, mwstart, mintys, clust \& llygoden, llysiau Ifau, gold mari, dail poethion, persli, palat y wàl, llygad Ebrill, neu lysiau y peil, rhos Mari, rhiw y gerddi, sage, sage gwylltion, neu chwerwlys yr eithin, Solomon sea!, juniperberries, ysgall bendigaid, neu gribau Mair, tyme, dail ysgyrf, smallage, triagl y moch, sef tormentile, tansi, a'r wermod. Dyma enwau y thai poethion yn eu natur, pa rai sydd j'w defnyddio at ddoluriau sydd yn magn trwy oerfel:

Yn nesaf cewch y rhai oerion yn eu natur, fel y canlyn :-

Arac, neu droed yr $\hat{w} y d d$, sef llysiau'r fain wyllt, y ben galed, dail llwyn hidl, llysiau'r ychen, lili'x gerddi, lili y dwfr, llygad y dydd, dail y rhoccos, rhosynau cochion, pwrs y bugail, dant y llew, cwmphri.

Dyna i chwi enwau y dail sydd oerion yn eu natur, fel pan y byddo arnoch eisiau oeri'r ystumog neu y gwaed, neu ryw ranau tu mewnol neu allanol, gellweh yn hawdd ei wneuthui gyda rhai neu $y$ cwbl o'r dail byn. Ac os bydd y gwaed yn rhy oer, ac eisieu ei wresogi, gellir ei dymheru gyda'r rhai poethion a enwyd. Niddywedaf ddim yo ychwaneg am natur y dail. Myfi a adawaf i chwithau chwilio allan natur eich dolur eich hunain, ac yna gellwch yn hawdd, trwy ddarlen y ddalen hon weled natur y dail, a thrwy hyny drefnu y fedayginiaeth yn gyfaddas i'r clwyf.

Angelica, neu lysiau yr Angel, liysiau'r Ysgyfaint. Y mae y dail rhagorol hyn yn tyfu memn gerddi, 
am byny mi thinaf y darllenydd a'u dull. Maent yn yn eu llawn nerth yn mis Gorphenaf, a'u hâd yn addfedu yn mis Tachwedd. Y mae y dail rhagorol hyn yn gorclifygu gwenwyn, trwy eu bod yu cryfhau y galon, ac yu ei chyunorthwyo yn ei erbyn, ac yn erbyn y pla yr un modd, trwy gymeryd haner dram o'r gwreiddyn, wedi ei bowdro, a'i gymeryd mewn triagl, a gorwedd in y gwely a chysgu. Y mae pob rhan o'r ddeilen hon yn llawn rhinwedd, ond ei gwreiddyn; y maent yn dda $i$ wellhau yr holl ddoluriau sydd yn dyfod i mewn i'r corph trwy oerfel; nid aml y maent yn fiaelu symud ymaith y cryd, trwy eu cymeryd ddwy neu dair gwaith o thaen y ffit, haner dram o'r gwreiddyn, neu ddeg gronyn o'r hadau sydd yn ddigon i'w gymeryd ar unwaith, a phowdr o'r gwraidd wedi ei gymyggu â mêl sydd dda rhag diffyg anadl, a'r pesychu, a phob afiechyd arall yn y frest; os bydd wedi magu drwy oerfel, y maent yn helpu poenus ofidiau $y$ colic a'r grafel, ac yn helpu merched yn ell harferiad misol. Ond cofiwch gadw y corph yn dymherus. Y maent yn helpu y Fam a'r Ysplin, ac yn gosiwng chwydd tufewnol; a'r sug trwy ei dropio i'r llygad sydd yn ei sirioli, ac y mae yn helpu y clyw drwy ei ddyferu i'r glust, a'i roddi yn nhwll y dant a ddofa y ddanodd; y gwraidd yn bowdr a’i wneud yn blastr $\hat{a}$ phŷg, a'i roddi ar frathiad ci cynddeiriog, neu ryw beth gwenwynig arall a'ugwellhâ yn fuan Nid ydwyf am ymhelaethu yn bresenol arnynt, o herwydd y mae hir-ddydd haf yn rhy fychan i ysgrifenu eu rhinwedd yn gyflawn.

\section{Balm, neu Bôm.}

Mae y dail hyn yn tyfu mewn gerddi, yn ad- 
nabyddus trwy'r holl wledydd. A'u rhinwedd fel $y$ canlyn. Y mae y dail hyn yn cryfhau y rhanau tumewnol yn rhagorol, sef y galon, y lle mwyaf angenrheidiol. Fe fyddai yn dda genyf glywed fod suryp wedi ei weuthur o'u sug a siwgr coch, yn cael ei gadw yn nhai holl foneddigion ein gwlad, fel ag y gallont ei gyfranu yn rhad ir tylodion afiachus. Y mae yr Arabiaid yn derchafu eu rhinwedd ir graddau uchaf. $Y$ maent yn foddion ir meddyliau a'r galon lawenhan, ac yo iachau y rhai sy'n dueddol o gael ffitiau yr eu cwsg, ac yn gyru ymaith holl feddyliau trafferthus, y thai sydd yn codi oddiwrth y melancoli. Ac y maent yn dda rhag brathiad ci cynddeiriog, trwy en berwi mewn gwin, yfed y gwin a rhoddi y dail yn allanol ar y lle; ac y maent hefyd yn dda $i$ ferched i'w berwi, ac eistedd arnynt yn glds uwch ben eu hager, $i$ ddyfod g'u cyrsiau misol i lawr. A's dail hefyd sydd dda wedi eu cynhauafu a'u powdro a'u cymysgu â mêl, rhag diffyg anadl a phesychu. Gellwch eu cymeryd wrth eich deall eich hunain : os yn leision, gwasgwch eu sug, a berwch ynghyd a siwgr coch; ac os wedi eu sychu, berwch mewn dwfr a gwin, ac yfwch w honynt i wellhau yr holl ddoluriau a enwyd uchod.

\section{Betony, neu Gribau St. Fraid.}

Dyma un o'r dail a ddylai gael ei chadw yn mhob teulu; y maent yn tyfu mewn lle cysgodol, ac yn blodeuo yn mis Gorphenaf. $Y$ mae un Physygwr wedi ysgrifenu llyfr mawr a helaeth ar rinweddau y dail hyn, ac yn mysg ereill o'u rhinweddau, y mae yn cadarnhau eu bod yn cadw yr iau a'r corph oddiwrth y pla; ac hefyd y maent $y_{n}$ 
cynorthwyo y rhai ac y mae eu hystumog yn rhy wan i gyual eu bwyd, ond yn ei dafu allan; ac $y$ maent hefyd yn cynorthwyo y rhai ag y mae eu bwyd yn suro yn eu hystumog, ac y maent yn gwella y clwyf melyu, a'r ffitiau confylsion, a'r polsi, a'r dropsi, ac yn dda $i$ wellhan y gowt a'r gewynau; ac y maent yn gwellhau y rhai sydd yn cael eu mynych flino gan boen yh y pen, trwy eu berwi mewn dwfr ynghyd a pennyroyal, a'u hyfed yn y bore, mae yn lladd y llyngyr, ac yn gwellhau y cryd, trwy eu cymeryd yn fynych o flaen y fit, ac yn tori poen yn y cefn a'r ystlysau, a'r holl ranau tufewnol, ac y maent yn cynnorthwyo y cyrsiau misol a ffitiau'r fam, ac yn esmwythau ffitiau'r gareg, a'r grafel.

Gellwch eu berwi mewn diod fain, neu win a dwfr, a'u hyfed yn fynych ac yn helaeth, wedi ei dwymno yn glaiar $i$ wellhau yr holl ddoluriau a enwyd uchod; ac wedi eu sychu, gellwch eu powdro, a'u cymysguâ mêl rhag pesychu a diffyg anadl, a rhag y consumsion ar yr ysgyfaint, a phob rhyw afiechyd arall yn $y$ frest; ac nid ydynt ddim llai eu heffaith mewn pethau allanol, sef briwiau hen a newydd, trwy eu mynych olchi â'u sug; ac y mae ychydig o'u sug yn dda i'w ddiferu i'r llygaid pan fyddo yn ddolurus. $Y$ mae y dail hyn yn rbagorol ac yn anghydmarol o dda, a dylent gael eu cadw yn mbob tỳ trwy y flwyddyn.

\section{Burdoc, new Cacamwci lleiaf, Cedorwrach.}

Y maent yn fwyaf neillduol i ferched, trwy rinwedd y dail a'r hadau y gellwch dynu'r groth y ffordd y mynoch, os bydd hi yn syrthio i lawr, 
gellwch wneud plastr o'r dail neu'r had, a'i rodd ar goryn y pen, a hyn a'i cwyd i fynu; ac os bydd y groth yn codi i fynu, ac yn eich mygu megys gwynt y fam, rhoddwch y plastr wrth wadnau eich traed, ac os byddwch yn dewis cadw y, groth yn ei lle, rhoddwch y plastr wrth ei bogel. Dyma un ffordd dda i sefydlu y plentyn yn y groth. Cymerwch un dram o'r gwraidd wedi eu powdro ar un waith rhag poeri gwaed; cymerwch lonaid llwy fwrdd o sug y dail mewn hen win, a hyny'n fynych, rhag brathiarlau nadroedd a chwn cynddeiriog, a churwch y gwraidd, a chymysgwch $\hat{a}$ halen, a rhoddwch yn allanol wrth y brathiad; a chymysgwch Iwyaid o'i sug mewn mêl, a chymerwch yn fynych rhag y grafel, neu'r had wedi eu powdro, a'u cymeryd mewn gwin gwyn, yn allanol, fel y canlyn, curwch y dail, a rhoddwch hwynt yn blastr poeth wrth y gewynau pan ag y byddont yn boenus, neu yn cwtogi; curwch y dail ynghyd a gwyn wy, a rhoddwch yn blastr ar losg tân, yr hyı a ddiffydd y tân ac a'i gwella yn hollol iach. Gellwch gymeryd yr had a'r gwraidd yn dufewnol wrth eich deall eich hunain. Pan ag y siaradwyf am ddail a rhyw enbydrwydd yn eu canlyn, myfi a roddaf swm dognau ar lawr.

Bey leaves, neu Ddail Cwru.

Nid oes eisiau dweud dim am eu dull, ond am eu rhinweddau, fel y canlyn,--y maent yn gwella y clwyf melyn, a'r dropsi, ac y maent hefyd yn cynnorthwyo y merched yn eu harferion misol, ac wedi genedigaeth plant, ond iddynt beidio a'u cymeryd cyn bod ar ben eu hamser, ac y maent liefyd yn 


\section{8}

chwalu gwynt, ac yn gwellhau y grafel, ac yn gwellhau ffitiau y Fam, ac yn lladd y llyngyr.

Y tfordd i'w cymeryd:-Gellwch eu cynhauafu a'u powdro, a chymeryd ychydig o honynt ddwy neu dair gwaith yn y dydd, ar win, rhag y pethau a enwyd uchod; os rhag pesychu a diffyg anad, "cymysgwch hwy mewn mêl, a chymerwch ef o hono, ac os bydd y dail yn leision, berwch hwy mevu rhyw beth cyfaddas, a rhoddwch fêl ynddynt, a chymerwch hwynt yr un modd.

\section{Blue Bottle, neu y Ben Galed.}

Maent yn tyfu yn mysg yr $\hat{y} d$.-Dyma ddail loer yn eu natur, ac y mae y powdr o'r dail sychion, '’u cymeryd ar fêl, yn rhagorol o dda i rai sydd wedi cael codymau, a'u briwio oddi fewn, ac i atal swaedu o fewn, trwy eu cymeryd mewn dwfr wedi perwi cumphri ynddo; ac y maent hefyd yn dda i wellhau brathiadau pob math o ymlusgiaid gwenwynig, a'u sug sydd yn iachau pob briwiau newydd, ic yn eu prysur groeni.

\section{Broom, neu Fanadl.}

I dreulio amser am eu dull, nid yw o ddim lleslad, gan en bod mor adnabyddus trwy y wlad, ac in difetha yr holl dir lle maent yn tyfu. Eu rhinveddau sydd fel y canlyn:-mae eu had, wedi eu howdro, a'u cymeryd mewn gwin, yn gweithio $\mathrm{yl}^{\circ}$ (nwmer dyfrllyd i lawr o'r corph, sef y dropsi a'r wowt, a phoen yn y cymalau ac yn y cefn, ac i iluellhau y cluyf melyn, ac i'w berwi mewn dwfr i Hchi y coesau chwyddedig: ac os berwch hwynt Hewn oil, hwy a fyddant yn llawer iawn gwell. A 


\section{4}

dyma yr anffaeledig beth i ladd yr holl lau yn.y pen, a'r holl leoedd ereill hefyd.

\section{Buck's horn plantcin, neu Ddail llwyn hidl.}

$\mathrm{Y}$ dail hyn wedi eu berwi mewn gwin, a'u hyfed, a rhoddi $\mathbf{y}$ dail yn allanol ar y lle, sydd yn iachau brathiadau nadrodd, ac y maent hefyd yn iachau dolur y gareg, ac hefyd yn iachau y rhai sydd yn taflu eu bwyd i fynu, ac yn atal gwaedu o'r genau a'r ffroenau, a'r clwyf gwaed, a'r rhai sydd yn gwneuthur dwfr gwaedlyd, trwy eu berwi mewn cwrw, neu win, a'u hyfed nos a bore; y maent yn iachau pob math o ddoluriau yn y llygaid.

\section{Colts foot, neu ddail carn yr Ebol.}

Y maent yn adnabyddus i bob dyn, yn eu nerth. - Ionawr hyd Fawrth; y maent yn rhagorol o dda rhag pesychu a chaethiwed, ond y dail wedi eu sychu sydd oreu i rai a fyddo yn pesychu ac yn poeri llawer, i'w cymeryd megis tobacco a'u smocio; ac: y maent hefyd yn dda i ostwng chwydd, ac y maent yn dda hefyd rhag tân eiddew, trwy eu gowneud yn eli â bloneg, ac yn dda hefyd rhag y peils, triwy eu berwi mewu dwfr, a rhoddi cadachau poethion an y lle. Y ffordd i'w cymeryd yw eu berwi mewn dwfr, a rhoddi yuddynt fêl a siwgr coch, a'u cymeryd wrth eich angeu.

\section{Celandine, neu lym y llygad.}

Mae y dail hyn yn anghydmarol o dda trwy wasgu eu sug a'i gymysgu ầ mêl, i'w roddi mewr llygadau clw'yfus, ac i wisgo'r bysen oddiar y gan- 


\section{5}

wyll. Hyn a allaf gadarnhau fy hun, trwy ei dreio mrywiol weithiau, a phob tro gyda llwyddiant. Ac ya mbellach, berwen y dail âg ychydig had mis, mewn gwin gwyn, ac yfwch o honaw yn ynych, fe wellhâ'r iau, y bustl, a'r clwyf melyn, ic y mae yn dda rhag $y$ dropsi; os powdrwch givraidd a'u rhoddi mewn dant a thwll ynddo, fe a syrth allan; a'u sug wedi ei gymysgu $\hat{a}$ flywfr brwmstan, a wellhâ'r ymgrafu, ac a gliria'r croen bob drwg liw.

\section{Cwmphri.}

Dyma ddail oerion, yn y trydydd gradd, y maent 'n giellhau y rhai sydd yn poeri eu gwaed, a'r hai sydd yo gwneuthur dwfr gwaedlyd; a'r gwraidd, vedi eu berwi mewn dwfr neu win, a'u hyfed, sydd in dda at bob math o friwiau tufewnol, ac yo ynnortheyo rhai $i$ boeri yn esmwyth pan fyddont in methu cael flems i fynu; y maent yn atal $y$ yrsiau misol pan y byddant yn wy helaeth, a ywynion yr un modd, trwy eu berwi mewn gwin och, ac yfed y gwin, neu gymeryd powdr o'r wraidd ar win. Ac yn allanol curwch y dail, a hodawch hwynt ar friwiau newyddion, a hwy a groenant yn fuan, ac nid oes eisiau mo'u gwell i rai fyddo wedi tori en lleingig, trwy yfed powdr honynt ar win coch, a rhoddi'r dail yn blastr llanol ar y lle; y dail hefyd sydd dda i'w givneuthur n blastr neu bowltis, a'i roddi ar fronau merched an fyddo gormod o laeth ynddynt.

\section{Camomile.}

Mid yw yn angenrheidiol $\mathrm{i}$ dreulio amser gyda $y$ hai hyn, gan fod eu rhinwedd mor adnabyddus yn 


\section{6}

cich plith am chwalu pigiadau, a thori poen yu y ystlysau, ac i chwysu yn nechreu y doluriau, : maent yn gyffredin yn dda mewn chwys i esmwyth hau'r corph o goryn y pen hyd wadn y troed, ac : maent yn dda i wellhau y cryd, trwy ell byfed yi gryf yn nechreu y fitit, a chwysu ar ei ol, ac yn du fewnol y maent yn esmwythau poen yn y bol, : colic, a'r grafel; y ffordd oreu yw cymeryd blodau, a'u gwnend y! bosel gyda diod frag thriagl, a chwysu i dori pigiadau; ac y maent yu dyfod a'r cyrsiau misol i lawr ar ferched, a'u sug wedi eu berwi vnghyd a'u blodau mewn gwil gwyn, sydd dda rhag y clwyf melyn a'r dropsi.

Yn allanol, y maent yn dá igryfhau y gewynau trwy eu golchi â hwynt yn boethion, ac nis gellucl gael gwell deilen na hon at bob math o oerfel yi yr aelodau.

Cymerwch eu sug ynghyd a siwgr rhag y 'splin y maent yn rhagorol o dda rhag dolur y gareg; hyn a'i profa, os cewch gareg wedi ei thynu oddi wrth ddyu, a'i rhwymo i fynu mewn camonile cewch weled y todda, yn fuan hefyd.

\section{Dwarf Eider, neu Ysgaw Mair.}

$\mathrm{Y}$ mae y cangenau ieuangc sydd yn tarddu alla o'r rhai'n yn dda rhag y dropsi, trwy eu berw mewn broth a'i yfed; ond os craswch y rhisg cano a'u berwi mewn dwfr, a'u hyfed, y maent yn llawe iawn cryfach, ac yn fwy awdurdodol yn erbyn dropsi; y mae y gwraidd hefyd yn dda rhag brathia neidr a chi cynddeiriog, trwy eu gwneud yn blast a'u rhoddi ar y lle; a'r dail sy'n dda i'r merche i'w berwi mewn dwfir, ac eistedd uwch ben yr age 


\section{8}

ifeddalhau caledrwydd y fam, ac i ddyfod a' cyrs iau misol i lawr, neu berwch eu brigau mewn gwin, ac yfich o hono i'r un dyben; ac y mae y sug, triy ei ddiferu i'r clustiau yn esmwythau eu poen, ac os berwch y brigau mewn gwin, a'i yfed, chwi a'i canfyddwch yn dda thag y grafel, a dwfir wedi ei ddystillio o'u blodau sydd yn harddu y corph, ac yn tynu lliw yr haul a phob rhyw anharddwch oddiar y croen.

\section{Dandelisn, neu Ddart y Llew.}

Eu rhinwedd sydd fel y canlyn,-Berwch eu gwraidd mewn gwin gwyn, os gellwch ej gael, onid ê mewn dwfr, ac yfwch o hono rhag y grafel a'r clwyf melyn, a'r splin, ac yn enwedig, peidied $y$ rhai sydd mewn darfodedigaeth ei anghofio, o herwydr ei fod yn rhagorol o dda jddyut i'w yfed yu ifynych; ac y maent yn caniatau esmwythdra a chwsg esmwyth i'r rhai sydd yn methu ei gael o herwydd y gures yn eu cyrph. Y mae y Ffrancod a'r Dutch yn eu bwyta yn spring y flwyddyn, i yadw eu cyrif mewn jechyd; ac os edrychwch ychydig yn mbellach, gellwch weled yn eglur fod physygwyr gwledydd ta amor a llawer Jlai o hunanbldeb ynddynt nag sydd ynom ni yn y wlad hon, a'u bod yn liawer mwy ymdrechgar i ddangos rhinxeddau y dail i'w cydgreaduriaid.

\section{Eringo, neu Gelyn y Mor.}

Y maent i'w cael ar hyd glan y môr, agos yn nhob gwlar sydd yn terfynu ar y môr. Y maent n eu natur yn boeth $\mathrm{y}^{\mathrm{y}} \mathrm{y}$ r ail radd, ac yn cryftau 


\section{8}

yr ysbrydoedd; os berwch y gwraidd mewn gwin, a'u hyfed yn fynych, y maent yn gwellhau'r frest, yr iau, yr gsgyfaint, y splin, y grafel, y clwyf melyn, y dropsi, poen yu y cefn, y llwynau, y colic, ac yu gwellhau cyrsiau y merched. Mae yn gofyn eu hyfed yn helaeth nos a bore am bymtheng niwrnod rhag y doluriau uchod. Ac os mewn dwfr y berwch hwynt, rhaid ei ferwi ar dân araf, yn hir, a chauad clos ar y llestr, $i$ ddal yr ager i mewn, a rhoddi ynddo chwarter pwys o siwgr coch at bok chwart i gydferwi âg ef, a'i botelu at eich gwasanaeth. Ac yn allanol y maent yn gwellhau y manwynau, trwy guro'r gwraidd a'u rhoddi yu blastr at y lle.

Eye bright, neu y Dorfagl, neu, Lygad siriol.

Fe'u gelwir fel hyn oherwydd sirioldeb y blodau ac hefyd olerwydd eu rhinwedd at y llygaid. maent yn tyfu mewn gweirgloddiau gwair, a thyndi trwy'r holl wledydd. Pe byddai y dail byn yn cae eu defnyddio mor fyuych ag y maent yn cael ex hesgeuluso, hwy a ddifethent waith y riai sydd yn gwneud yspectols; y sug neu y dwfr a ddistilli o'r dail sydd dda i'w diferu i'r Iygaid clwyfus; a yfed y dail fel tea, nou wedi eu berwi mewn broth a'i yfed yu fynych, a gryfhâ y golwg pan y byddo' pallu trwy henaint, neu ryw achos arali: maen hefyd yn cryfhau g synwyrau gweinion, trwy en berwi mewn diod frâg, a'u hyfed yu fyuych.

Hox's Gloves, neu Fenyg y Lluynoy, nex delail Bysedu Cochion.

Fet ag y mae y dail hyn yn admabyddus trwy y toll wledydd, ni flinaf y darllenydd â'u dull; maen 


\section{9}

yn tyfu yn fwyaf cyffredin mewn tir diffrwyth, ac ochrau cloddiau. Mae y dail hyn yn glanhau briwiau yn raddol, gyd a'r tynerwch mwyaf. Mae yr Italians yn eu mynych ddefnyddio i lanhau ac i groeni briwiau newydd, trwy guro y dail a'u rhoddi arnynt megis plastr, a'u sug hefyd sydd dda i lanhau hen friwiau drewedig, eu sychu a'u croeni. Ond y mae amrywiol o ddysgedigion yn eu cymell yn dufewnol, i weithio'r corph i fynu ac i lawr rhag y dropsi, ac amrywiol ddolurian ereill. Ond ni leiciwn i mo'u cymeryd, am hyny ui's gallaf eu cymhell i ereill; ond byn a ddywedaf, trwy eu profi fy hun, rai cannoedd o weithiau, na thywynodd haul erioed ar well deilen i'w rhoddi mewn powlis at hen friwiau a newydd, ac at yr hyn a elwir yn gyffredin clwy'r brenin, neu y manwynau. Hyn a brofais fy hun, rai ugeiniau o weithiau, a phob tro yn llwyddiannus: a myfi a allaf ddywedyd wrthych nad oes mo'u gwell i'w gwneuthur yn eli gyca bloneg, i wella crach mewn pen.

\section{Fern, neu Redyn.}

Mae dwy ryw o'r rhai hyn, fel y canlyn, sef yr wrryw a'r fenyw-mae y fenyw yn tyfu yn uwch ac yn fwy bylchog; ond y mae eu rhinwedd yr un effaith yn gymwys; y mae eu giwaidd wedi eu morteru, a'u berwi mewn dwfi, a'u liyfed yn forfeuol, yn lladd dau fath o lyngyr, y llydain a'r crynion, ac yn gwellhau caledrwydd a chwydd yr ;sblin, eu gwraidd wedi eu powdro mewn bloneg, ydd yn gwneuthur eli gwerthfawr i iachau briwiau, aeu bigiadau drain gwenwynig; ac os shoddwch eu wowdr mewn hen dyllau dyfrllyd a drewedig, hwy 
a'u hiachânt ac a'u croenant yn fuan. Dymunaf ar i'r merched beidio a'i gymeryd yn dufewnol; ond y mae i'r meibion yn ddiberygl.

\section{Fennel, neu Ffanigl.}

Maent yn dda i dori gwyut ac i'w chwalu, ac yn dda rhag y grafel, ac yn help i dori'r gareg; a'r dail wedi en berwi mewn barley water, neu ddwir haidd, sydd yn dda i ferched i chwanegu eu llaeth. a'i wneud yn fwy iachus i'w plant, ond iddynt ei yfed yn lled aml; a'r hadau, wedi en berwi mewn gwin, sydd dda i iachau brathiad neidr, neu i rai wedi bwyta dail gwenwynig, neu gegyd, neu ryw beth o'r cyfryw. Mae yr hadau i'w cael yn siop y Druggist drwy y fiwyddyn; y maent hefyd yu dda trwy eu berwi mewn pottes, $\mathrm{i}$ wnend $y$ rhai sydd dueddol i fyned yn rhy dewion yn fiwy teneu.

\section{Ground Ivy, neu Eiddew y ddaear.}

Mae'r dail hyn yn rhagorol o dda i iachau briwiau yn dufewno!, sef yr iau a'r ysgyfaint, ac amryw bethau o'r fath, ac y maent yn esmwythâu yr holl boen yn y bol a'r ystlysan, ac yn chwalu gwynt ac yn esmwythâu y colic, ac y maent yn dda rhag gwenwyn a'r plấ, a'r melancoli, a'r grafel, a'r gowt yn y glinian a'r traed. Gellwch eu berwi mervn dwfi, a'u byfed rhag yr holl ddoluriau uchod, neu symeryd eu sug mewn gwin gwyn a'u hyfed, ond am ba faint $i$ gymeryd o honynt, neu yr amser $i^{9} w c y-$ meryd, nid yw yn angenrhaid colli amser, am etu bod yn hollol ddiniweid; a phe b'aent ddail peryglus, mi a'ch rhybyddiwn chwi yn helaeth, ond mi a'i 


\section{1}

cyfrifais yo ddoethineb $i$ adael y rhai hyuy allan o's llyfr.

Ond yn allanol y maent gn dda i'w berwi, a rhoddi ynddynt fêl ac alwm i olchi'r genau a'r gwddf pan fyddont yn ddolurus; y maent yn prysur iachau briwiau newydd, trwy eu cnocio, a'u rhwymo arnynt; a'u sug, wedi ei ferwi, ag ychydig fêl a verdigrise, sydd dda $\mathrm{i}$ olchi hen friwiau drewedig, ac y maent yn dda i wellhau'r crafu a'r crach, a phob toriad allan yn mhob rhan o'r corph; ac y mae eu sug yn dda i dori mewn clustiau. Cymerwch o'u sug ac; o celandine, ac o lygad y dydd, yr un faint o bob un, a rhoddwch yuddo siwgr dwbl refined, y mae yn rhagorol o dda $i$ wellhau y llygad clwyfus a dyfrllyd, ac i wisgo y bysen; ac y mae mor dda i anifeiliaid ag $i$ ddynion.

\section{Gooseberry bush, neu ddail Gusberins.}

$Y$ mae y gwsberins cyn iddynt fod yu addfed yn dda i'w sgaldio, neu eu crasu mew pobdy i's rhai sydd wedi colli eu ystumog; ac y maent yo rhagorol o dda $i$ dori blyss ar ferched pan fyddont yn feichiog. Gellwch eu cadw drwy y flwyddyn, trwy eu presarfio â siwgr, a'u cymeryd wrth eich angen; ac $y$ maent yn dda pan y byddont wedi addfedu i'w bwyta, i oeri yr iau a'r ystumog pan y byddont yn rhy boeth. A'r dail pan y byddont yn ieuange ac yn dender, sydd dda i'w hyfed thag $y$ grafel, ac yn allanol i olchi chwydd coch a chaled.

\section{Horownd.}

Berwch y dail syahion a'r hadau yughyd a mel, ac os bydd y dail yu leision, cymerwch eu sug, ac 


\section{2}

yfwch o hono yn lled fynych rhag pesychu a diffyg anadl, a'r darfodedigaeth, ac $y$ maent yn dda mewn hir a phoenus drafael, i'w cymeryd yn y ffordd uchod, y maent yn dda i rai fo wedi cymeryd gwenwyn, neu eu pigo, neu eu brathu gan ryw ymlusgiaid gwenwynig, a snyfio eu sug i'r froenau sydd yn gwellâu'r clwyf melyn, a’i gymysgu âg oil rhosus, a'i ddiferu i'r glust, sy'n tori y boen yno; ac y maent yn gweilhau y frest, yr iau, yr ysgyfaint, a'r ysplin, trwy eu hyfed yn fynych; cymeryd ychydig o'u powdr bob boreu sydd yn liadd y llyngyr yn y bol, a churo y dail yn leision ynghyd a bloneg; sydd yn gwellhau brathiadau cwn, a phigiadan drain.

\section{Hyssop.}

Berwch isop, ac ychydig ryw' gerdid a mêl, ac yfwch o honynt rhag pesychu, diffyg anad, a chrygni yn y fiest a'r gwddf, a churwch y dail ynghyd $\hat{a} g$ ychydig siwgr, a rhoddwch ar friwiau newydd, ac fe a'u hiachâ yn fuan.

\section{Holy Thistle, neu Ysgall bendigaid.}

$Y$ mae yn debyg y rhoed yr enw hwn arnynt gan ryw un a phur ychydig o sancteiddrwydd yuddo ef ei hun, eu whinwedd sydd fel y canlyn:-Y maent yn gwellhau ysgafnder mewn pen, a'i clwyf melyn, ac yn puro y givaed, a gw ynebau cochion a'r ymgrafu, penaddynod, y pox, a brathiadau cwn cynddeiriog, ac hefyd i gryfhau'r cof; y fordd i, $w$ cymeryd,powdrwch y gwraidd, a clymerwch o honynt mewn gwiu, neu ryw beth cyfaddas, dair gwaith yu y dydd. 


\section{3}

Honey suckle, neu ddail Grwinwydd.

Nid wyf yn gwybod am ddim gwell deilen at $\mathrm{y}^{\mathrm{r}}$ asthma, neu hen besychu, ac hefyd y mae yn cymeryd ymaith holl afiechyd y 'splin, ac yn gwellhau y grafel, ac yn cyunorthwyo merched wrth esgor, ac y mae yn iachau y clymau gwythi, a'r fritiau confylsiwn, a phob rhyw anechyd sydd wedi tarddu trwy oerfel. Y ffordd i'wcymeryd, curwch y dail yn nghyd a'u blodau, a gwasgwch allan eu sug, a chydferwch hwynt â siwgr coch, a chymerwch o hono lonaid llwy fwrdd dair gwaith yn y dydd, rhag yr holl ddoluriau a enwyd uchod; os na fydd y dail i'w cael yn leision, berwch ynghyd a mêl, ac yfwch o hono. Ac os ewgllysiweh wneuthur enaint o honynt i lanhau eich crwyn, ac i dynu lliw yr Haul, curwch y dail a'r blodau, a berwch hwyot newn bloneg, ac irwch eich humain âg ef.

\section{Motiler-wort, neu Lysiau'r fam.}

Y maent yn tyfu mewn gerddi; y mae amryw o honynt, ond y maent oll o'r un rhinwedd; nid oes dim guell dail yn tyfu $i$ hel melancoli o'r galon, ac i'w chryfhau, a'i gwneud yu llawen. Geilwch wneuthur surop o honynt, a'i botelu at eich gwasanaeth, cewch addysg yn y llyfi i weud byny; ac heblaw hyny, y maent yn gwheuthur gwragedd yn famaethod llawen wrth ddwyn plant i'r byd, ac yn sefydlu y groth fel y dylai fod; y maent yn dda pan fyddo'r galon yn curo ac yn dueddol i wasyfauon, yn nghwsg ac yn effro, ac y maent yn dda rhag ffitiau, neu wynt y fam, rhag y grafel, ac it ladd llyngyr, ac yn gwellhau y clymau gwythi, a 
ffitial confylsiwn: cymerwch ell powdr mewn gwin, a berwch ef, ac yfwch o hono yn fynych.

\section{Maiden hair, neu Wallt y Forwyn.}

Berwch hwynt mewn gwin a dwfr, a chymerwch - hono rhag y pesychu, y diffyg anadl, y clwyf melyn, dolur yr ysplin, ac at atalfa'r dwfr, ac i dori y gareg; berwch y dail mewn oil camomile, y maent yn dda i ostwng chwydd, y maent yu dda i'r thai fo'n colli eu gwallt, ond eu berwi mewn gwin a sweet oil, neu ulew pêr, a rhwbio y gwallt.

\section{Marsh-mallows, Hocys, neu y Rhocos.}

Gwraidd $y$ dail yma sydd dda i'w berwi mewn broth i ryddhau ac $\mathrm{i}$ dymheru y corph, pan y byddo yn dueddol o fod yn rhy rwym; rloddwch eu sug unewn gwin i wraig a fyddo ar ben ei hamser, a hi a gaiff fyned i'w gwely yn fuan, a diberygl; a phan y byddo y merched yn brin o laeth yn eli bronau, hwy a gant helaethrwydd, trwy fynych ddefuyddio'r dail hyn; a llonaid llwy firdd o'u sug bob bore sydd ragorol o dda $\mathrm{i}$ gadw y corph mewn iechyd, ac hefyd i'r rhai sydd yn dueddol i daflu eu bwyd; y gwraidd wedi eu morteru, a'u grwneud yn bowltis gyda blawd haidd a mêl ac oil rhosus, y maent yn dda $\mathrm{i}$ ostwng chîyydd; cofiwch ferwi eu gwraicld mewn llefrith a chwrw, ac yfw ch yn helaeth o hono; a hyn gyda bendith Duw, a'ch gwna yn hollawl iach o's clwyf gwaed.

\section{Mustard.}

$Y$ maent yn rhagorol o dda $\mathrm{i}$ bawb ag sydd eisio puro'r gwaed a chryfhau yr ystumog, ac $y$ maent 


\section{5}

yn rhagorol o dda $\mathrm{i}$ hên bobl pan $\mathrm{y}$ byddont yn cael eu poeni gan ddoluriau a fyddo yn tarddu oddiar oerfel, a'u grrneud yn deneu ac yn oer. $Y$ mae y mwstard yn boeth yn y pedwaredd gradd, a thrwy hyny yn cysuro y natur oerllyd; gadewch i henaint wneuthur yn fawr o honynt, ond am yr ieuengtyd sydd a'u gwaed yn llawn gwres, nid oes arnylit ddim angen am danynt. Berwch eu had mewu gwin gwyn, shag y grafel a'r gareg; y maent yn lladd nerth gwenwyn yn y corph; gellwch eu cymeryd mewn cwrw, neu ar eich brechdan, neu gyda chig, rhag y dolurian a enwyd.

\section{Mouse-ear, neu Lygad y llygoden.}

Y mae y dail hyn yn rhagorol o dda rhag y clwyf melyn, trwy eu hyfed nos a bore, a pheidio yfed un ddiod arall am ddwyawr ar ei hol; y maent yn rhagorol o dda $\mathrm{i}$ wellhau ffitiau o ddolur y gareg, yr ysplin, ais dropsi, ac y maent hefyd yn dda rhag pesychu a diffyg anadl; gellwch eu berwi mewn dwfi, a rhoddi mêl ynddo, ac yfed llonaid cwpan dea o hono dair gwaith yn y dydd, i wellhau y doluriau a enwyd.

\section{Mug-wort, neu lysiau Evan,}

Y mae y dail hyn yn help mawr yn erbyn ffitian y fam a dolur y gareg; ac y mae eu sug yn help mawr i'r rhai sydd wedi cymeryd gormod o opium, a chysgu yn rhy hir. Ac y maent yn rhagorol o dda $i$ ferched, i'w berwi mewn dwfr, ac eistedd arnynt uwch ben yr ager. Y ffordd i'w cymeryd yn dufewnol,-gelliwch en berwi j'w hyfed; neu eu powdro, a'u cymeryd mewn mêl. Yn allanol. 
maent yn iachau chwarenau o amgylch y gwddf, trwy gymeryd eu gwraidd a llygad y dydd a bloneg, a'u gwneud yn eli: a thri dram o'u powdr mewn gwin, a'i gymeryd ar unwaith, a iachâ y gowt, trwy ei fynych gymeryd.

\section{Mari-gold, neu gold Mair.}

Y maent yn cryfhau y galon yn rhagorol, ac yn taflu allan y pla a'r frech wen, ac yn cadw y galon yn iach oddiwrth $y$ pla trwy eu berwi mewn shyw beth cyfaddas, a'u hyfed yn fynych yn allanol.

Gwnewch blastr o'r blodau wedi eu powdro â bloneg ag ystor a thurpentine, a'i roddi ar y galon mewn clefyd, sydd yn cadw y galon ac $y n$ ei chadarnhau yn erbyn clefyd.

\section{Nettles, neu Ddanadl poeilion.}

Hâd y dail poethion i'w cymysgu mewn mêl, sydd dda i'w cymeryd rhag pesychu a'r diffyg anadl, a chaethiwed yo y frest, ac i helpu codi y fflems i fynu, fel ag y gellir poeri yr afiechyd allan.

Berwch eu had mewn gwin gwyn, ac yfwch rhag y grafel a'r garreg, mae hyn wedi ei brot yn aml; y maent hefyd yu lladd llyngyr mewn plant, ac $y$ maent yn dda hefyd i iachau brathiadau cwn cynddeiriog, a'r rhai a fyddo wedi bwyta cegyd yn eu hanwybodaeth; rhag brathiad ci drwg cymerwch yn dufewnol, a chnociwch y dail gyda halen, a rhoddwch yn allanol ar y lle.

\section{Parsly, neu Bersly.}

Mae y dail hyn yn fwy perthynol i chwalu y grafel, ac i dori y garreg nag un peth arall; $y$ ffordd 


\section{7}

oreu i'w cymeryd yhag y grafel yw eu rhostio megis llysiau, a'u bwyta.

Mae'r cyngor fel y canlyn rhag y clwyf melyn a'r dropsi, a'r ffitiau, a'r gareg,-cymerwch had persli, a had franigl, a had anis, a had carwe, owns o bob un, ac owns a haner o wraidd persli wedi eu tori yn fân, a malu yr hadau, a rhoddwch hwynt oll mewn llonaid potel o win gwyn dros nos, a berwch yn y bore, mewn llestr pridd cauedig, hyd nes ileihau y drydedd ran o hono, ac yna ystraeniwch a photelwch, a chymerwch o hono bedair owns nos a bore, a pheidio yfed dim ar ei ol am dair awr.

\section{Pellitory of the wall, neu Paladr y wal.}

Powdrwch y dail a chymysgwch mewn mêl, rhag y pesychu a diffyg anadl, a chaethiwed yn y gwddf, a chymerwch dair owns o'u sug pan fyddo y dwfr wedi stopio, ac efe a gliria y grafel allan o'r chwysigen; y maent yn esmwythau ffitiau y fam a'r 'splin. A rhag y peils cnociwch $y$ dail a berwch mewn bloneg, a gwnewch eli o honynt, a rhoddwch ar $y$ lle, a'r dail wedi eu cnocio ar friw newydd, a'i rwymo i fynu am dri diwrnod, nid rhaid $\mathrm{i}$ chwi ddim arall wrtho.

Sug y dail hyn wedi ei buro trwy ei ferwi mewn mêl, cymeryd llonaid llwy fwrdd o hono yn y boreu, ddwy neu dair gwaith yn yr wythros, a'ch gwna yn hollawl iach o'r dropsi, os cymerwch mewn pryd.

\section{Pile wort, neu lygad Ebrill.}

Nid rhaid ond coni dyrnaid o'r dail hyn i fynu, yna cewch weled dull y peils yn eu gwraidd hwynt; 
nid wyf yn rhyfeddu dim eu bod wedi eu galw yn ddail y peils, berwch y dail a'r gwraidd, ac yfwch rhag y peils a chwarenau o amgylch y gwddf, yr hyn a elwir yn gyffredin clwyf y brenin; ac os bydd y peils yu allanol, gwnewch eli o'r gwraidd a bloneg, ac irwch y lle âg ef, ac yfwch y diiod yr un pryd; dyna un dirgelwch eto genyf, os gwisgwch y dail hyn neu'r gwraidd nesaf i'ch croen, y maent yn gwellhau y manwynau er nad yw yn cyfhwrdd â'r lle clwyfus, eto y mae, medd thai, yn gwella y clwyf; ond hyn a gadarnhaf $f$ fy hum, mai y dail hyn a menyg y llwyoog, yw y ddwy ddeilen oreu a welais i erioed at glwyf y brenin, trwy eu gwneud yn bowltis â bloneg; dyma y moddion $\hat{a}$ pha rai y gwellais ugeiniau wedi i bob modcion eraill ballu; ond fe allai y gwel rhai yn foddion rhy wael i'w dreio; ond cofia hyn, na wnaeth y Duw doeth lysieuyn erioed yn ofer.

\section{Rhos Mari.}

Y maent yn ddail mor rinweddol ag un sydd yn tyfu, i'w defnyddio'n dufewnol ac yu allanol, o berwydd trwy eu cynesrwydd, y maent yn cysuro holl ranau y corph sydd wedi eu niweidio trwy oerfel, yn enwedig y pen a'r ystumog; y maent yn dda rhag ysgafinder yn y pen, yu crythau'r ymenydd, ac yn codi yr ysbrydoedd; ac yn gwellhau y polsi sydd yn attal lleferydd, ac y maent yu givella cof gwan, ac yn crythau synwyrau, ac yu rhagorol dda i'r frest, pan fyddo oerfel wedi setlo ynddi; chwi a ellwch eu powdro, a chymeryd o'r powdr mewn gwin gwyn; neu eu distilio, neu eu berwi mewn dwfr, a'u hyfed ynghyd ag ychydig win gwyn, 
neu frandi. Y mae eu blodeu yn helpu y rhai sydd a'u golygon yn ddwl, trwy eli bwyta yn y boreu gyda bara a halen; ac os berwch yn y bore mewn dwfr, a'u hyfed, a cherdded yn ffast ar ol hyny, fe a'ch iachâ o'r clwyf melyn; eu sychu a'u smocio fel tobacco, sydd dda i'r rhai sydd yn poeri llawer; ac yn allanol y maent yn dda i'w berwi i olchi cryd cymalau oer, neu ryw afiechyd arall yn yr aelodau wedi magu drwy oerfel; y mae oil i'w gael o honynt yn siop y Druggist, pa un a wellhâ yr holl ddolur; yr un tufewnol a enwyd, gellwch ei gymeryd o bump i bymtheg tropyn, dair gwaith yn y dydd, fel y byddo yr oed a'r nerth, mewn llwyaid o win gwyn.

\section{Rheu, neu Ryw y gerddi.}

Eu defnydd mwyai yw gwellhau merched, yn eu harferiad misol; ond cymered merched beichiogion ofal am gadw oddiwrthynt rhag iddynt, nid yn unig niweidio eu hunain, a gwneud iddynt fwy o niwaid mewn un awr nag a fedr y physygwr goreu yn $y$ wlad feddyginiaethu mewn un fiwyddyn. Y maent hefyd yn dda rhag y grafel, i'w hyfed yn yr un modd, wedi eu berwi mewn rhyw beth cyfaddas, ac yfed llonaid cwpan dea o honynt yn y boreu, a bwy a laddant y llyngyr hefyd. Ac os cymerwch eu hadau wedi eu powdro a'u hyfed mewn gwin, hwy a wnant bob peth gwenwynig yn ddiniweid i chwi; ac ychydig ryw a chanomil, wedi eu berwi ynghyd, a'u cymeryd amryw droiau o flaen y ffit o grynu, a'i gwna yn gwbl iach; a'u sug, ynghyd a sug ffanigl a mêl, a bustl ceiliog, yr un faint o bob un, a'i ddiferu i'r llygaid, pan fo'r golwg yn pallu, hya a wellhaodd lawer. 


\section{0}

Red Rcse, neu Rosus Cochion.

Berwch y rhosus cochion mewn gwiu, i'w hyfed, rhag poen yn y pen, ac y maent yn dda i' $w$ berwi mewn grwin coch i'w hyfed yn fynych, i atal y misglwyf, neu ryw beth cyifelyb; yr hadau yn eu canol sydd yn ateb yr un dyben, trwy eu powdro, a'u hyfed mewu gwin; y maent hefyd yn dda rhag clwyf y gwaed, a rhag givaedu trwy y genau a'r tfroenau, a'r rhai sydd yn gwneud dwfr gwaedlyd; ac y maent hefyd yn crythau y galon, yr iau, a'r ysgyfaint, ac yn codi yr ysbrydoedd, ac os ydych yn dueddol o daflu eich bwyd, cymerwch ddail rhosus a dail mintys, a rhoddwch hivynt yn allanol wrth eich hystumog, a hyn a'i tawela yn fuan.

\section{Säe, neu Saigs.}

Mae y dail hyn yn dda i'r iau, trwy e! hyfed yn dea, neu ffordd arall, ac i fagu gwaed iach yn y corph, ac a maent hefyd yn dda rhag y grafel, ac y maent yn atal gwaed mewn pob math o friwiau, trwy eu golchi â hwynt; llonaid llwy fwrdd o honynt sydd dda i ferched, ${ }^{\prime}$ 'w givneuthur yn ffrwythlon, pan y byddont yn anmhlantadwy, ac: hefyd i'w cadw rhag misgario; rhag cyfogi, neu boeri givaed, cymerwch dair llwyaid o'u sug ynghyd a mêl, hyn a'ch gwellhâ yn fuan; maent yn dda rhag poen mewn pell, ac yn ysgafuhau, ac yn cyfodi y meddwl isel i fynu; cymeryd eu sug mewn dwfr cynes a mêl, sydd dda rhag crygni a phesychu; gellwch hefyd eu defnyddio gyda rhos Mari a gwywydd, a dail llyriad, a mêl, a golchi y genau a'r gwddf à bwynt, pan fyddont yn ddolurus. 
Sage wild, neu Saigs gwylltion, Chwerwlys yr eithin.

$Y$ maent yn lled debyg i'r lleill, ac nid oes eisiau mo'u gwell i chwysu, ac i ostwng chwyydd yn yr aelodau, trwy wneuthur defnydd o honynt yn dufewnol ac yn allanol, ac y maent yn dda i wellhau y pox, trwy eu mynych yfed, ac y maent yn anffaeledig fecidyginiaeth i'r rhai sydd wedi cael codymau, a thori gweithenau oddifewn. Yfwch yn fynych o hono, ac fe'ch gwellhâ yn fuan.

\section{Wood Sorrels, neu Surens y Góg.}

Maent yn rhagorol o dda i oeri ac i buro'r gwaed, ac yn allanol, ni's gellwch gael eu gwell i ostwng chŵydd coch caled, ac i'w oeri.

\section{Sorrel, neu Surens y Cûn.}

Y maent yn dda at bob dolur poeth, pan y byddo eisiau ei oeri. Maent yn oeri yr ystumog pan fyddo yn rhy boeth, ac yn dda yn y ffit boeth o'r cryd, ac ymhob peth y byddo eisiau ei oeri, yn dufewnol ac yn allanol; ac y maent yn dda $i$ dori syched, ac i ladd y llyngyr, ac yn cryfhau y galon wan. Berwch eu gwraidd mewn dwfr, ac yfwch o hono rhag y clwyf melyn, ac i dori y gareg, ac i chwalu y grafel; ac os berwch eu blodau mewn gwin gwyn, a'u hyfed, y maent yn dda rhag y clwyf melyn a'r du.

\section{Senicl.}

Y maent yu prysur wellhau briwiau newydd, ac yn gwellhau'r postwm, ac yn atal gwaedu yn dufewnol; ac ni welwyd erioed mo'u gwell i drlyn ac 
anifail pan fyddo dolur o'r ysgyfaint. Chwi ellwch en berwi mewn diod fain neu ddwfr, a'u cymysgu $\hat{a}$ mêl, a'i yfed wrth eich syched, rhag doluriau yn $y$ frest, yr iau, a'r ysgyfaint ; y maent yn dda i wellhau'r genau a'r gwddf, trwy eu berwi mewn dwfr, a'u cymysgu â mel, a golchi y dolur âg ef jn fynych; ac y maent yn iachau y rhai sydd yn thur dwfr gwaedlyd a chymysglyd, ac yn atal rhyddni yn y bol. A chofiwch nad ydynt ddim llai eu heffaith i'r rhai sydd wedi tori eu lleingig, trwy eu hyfed fel ag y dangoswyd, a'u rhoddi yn bowltis wrth y lle; ac y maent yn rhagorol o dda at bob achos lie y bydd eisiau sychu, yn dufewnol ac yn allanol,

\section{Solomon Seal.}

Y maent yn tyfu yn y gerỏdi, ac yn gyffredin yn adnabyddus yn mhob. lle; y mae eu gwraidd yn thagorol o dda at friviau ac archollion, a damweiniau allanol, ac i gall y briwiau newydd i fynu, ac i sychu yr hen, trwy eu powdro, a'u rhoddi yn y briwiau; y maent yn atal y terms yn y merched, pan y byddont yn rhy helaeth; ac y maent hefyd yn dda i feibion sydd yn gwaedu o'u ffroenau, neu o'r genau, neu wrth wneuthur dwfr, trwy eu hyfed yn dea gyda siwgr gwyn; ac y maent yn rhagorol o dda trwy eu gwneuthur yn bowltis i gryfhau esgyrn briwedig, neu gymalau gweiniaid. Ac fe'u cafwyd allan yn ddiweddar yn anghydmarol o dda i'w berwi mewn gwin, neu ryw ddiod arall, a'u hyfed gan y rhai sydd wedi tori neu friwio eu hesgyrn; ac nid yw eu heffaith ddim yn llai i'r thai sydd wedi tori eu lleingig, trwy eu hyfed, a rhoddi'r dail yn blastr ar y lle am naw diwrnod, heb ystyrio ond 
can lleied ag a ellir. A'r dwfr w'edi ei ddistillio oddiw rthynt, a hardda y croen, ac a dyna liw'r haul, ac a lánhẩ bob anharddwch arall; y mae yr Italians yn eu harfer i'rdybenion hyny yn fynych.

\section{Seurvy-grass, nei ddail Ysgyrfi.}

$Y$ maent yn rhagorol dda i buro y gwaed, yr iau, a'r ysplin, trwy gymeryd o'u sug bob bore yn y spring, mewn cwpanaid o ddiod frag; a'u sug hefyd sydd dda i'w rodidi yn mhob math o friwiau dyfrllyd a drewedig, ac hefyd i lanhau y croen oddiwrth bob math o anharddwch.

\section{Shepherd's purse, neu burs y Bugail.}

Y mae yn gwellhau y clwyf gwaed, ac hefyd yn helpu stopio gwaed mewn briwiau yn dufewnol ac yn allanol, ac yn dda i'r rhai sydd yn gwneuthur dwfr gwaedlyd, ac yn poeri eu gwaed; i stopio terms yn y merched, trwy eu rhwymo wrth eu harddyruau a gwadnau eu traed; y mae yn gwellhau y clwyf melyn, ac yn iachau tân iddwf, trwy wneuthur yn bowltis. A'u sug sydd dda i'w dropio i'r clustiau i'w sychu, ac i dori sŵn yn y pen. Nid yw y dail hyn i'w defnyddio yn dufewnol, ond yn allanol fel y dywedwyd uchod.

\section{Thyme, Teim.}

Y maent yn tyfu yn y gerddi : y maent yi rhag orol o dda, i gryfhau yr ysgyfaint; nid oes mo'u gwell yn tyfu i fendio y pâs ar blant, a diffyg anadl, a'i wneưd yn dea i blant, a'i felysu â siwgr candi, a’i roddi iddynt yn ddiod; ac y maent yn lladd y llyng- 


\section{4}

yr, ac $y$ maent yn dda $i$ sragedd i'w cymeryd pan fyddont ar ben eu hamser, i gael myned i'w gwelyau yn esmwyth ac yn fuan, ac hefyd y maent jn dyfod a'r ail i lawr; a pheth a ellwch ddymuno yn ychwaneg; ac y maent mor ddiniweid ag y gellwch eu cymeryd y ffordd y mynoch hel ofni perygl with eu cymeryd; gn dufewnol y maent yn cryfhau $y$ galon a'b ystumog, ac yn chwalu gwynt.

\section{Tormentile, Triagl y moch.}

Maent yn tyfu mewn lle coediog a chysgodol; fe ddywedir eu bod yn rhagorol o dda i stopio gwaed o'r genau, y ffroenau a'r ymysgaroedd, a'u sug wedi ei gymysgu $\hat{a}$ venus triagl, a gorwedd i chwysu, sydd yn anffaeledig feddyginiaeth $i$ rai wedi cael gwenwyn, ac hefyd yn nechreu clefyd, a phob math o bla, ac nid yw y dail wedi ea berwi mewn dwfs i'w hyfed yn ddim llai eu heffaith i fendio y pox; mae eu sug yn gwellau y cliryf melyn.

Ac yn mhellach, berwch mewn dwfr yn drwyth gref, ac eistedded y merched sydd yn dueddol i fisgario uwch ben yr ager, a'u traed yn y dwfi mor boeth ag $y$ gallont ei oddef; a phlastr o honynt wedi ei wneuthur â finegr, sydd yn ateb i'r un diben. Ychydig o'u powdr gyda sug dail llyriaid sydd fn lladd llyngyr ar blant; ac y maent yu dda i'r rbai sydd wedi cael codymau a thori eu leingig, trwy eu hyfed a'u rhoddi yn blastr ar y lle. Eu gwraidd a phaladr Yspaen, ac alwm, sydd yn dda i roddi yn nhwll $y$ dant $i$ wellhau $y$ ddanodd; ac $y$ maent yn rhagorol o dda rhag y peils, trwy eu hyfed, a'u gwneud yn eli o honynt gyda bloneg a'i ddefnyddio yuallanol; ac yn dda i'r merched i'w byfed i ystopio y terms misol. 
Vervain, neu lysiau yr hudol.

Maent yn tyfu mewn cloddiau ac ochrau ffyrdd, a thiroedd gwylltion ereill, ac yn blodeuo yn mis Gorphenaf. Y mae y dail yn rhagorol o dda rhag yr holl oerfel a'r afiechyd sydd yu berthynol i's. groth, ac y maent yn iachau y clwyf melyn, a'r dropsi, a'r gowt, ac yn lladd y llyngyr yn y bol; ac y maent yn cryfhau yr ystumog, yr iau, a'r ysplin, ac yn gwellhau y pesychu, a'r diffyganadl, ac yn dda rhag y grafel, a'r gareg; chwi a ellwch cu distillio, ac yfed $\mathrm{y}$ dwfi a ddistilliasoch oddiwrthynt syda mêl, neu eu berwi, a gwneuthur surop o honynt, a'i gymeryd wrth eich angren.

\section{Wermod.}

Ni thywyodd yr Haul erioed ar well deilen na hon i wellhau y clwyf melyn, ac y mae yn dda hefyd rhag y grafel, ac i ostwng chwydd yn y bol. Myfi a ddywedaf newydd da i'r tylodion nas gallant dalu i Feddygon, cymerwch flodau y wermod, a rnos Mari, dail drain duon, yr faint o bob un, a berwch hwynt ynghyd, ac ar ddiwedd y berwi, rhoddwch ynddo haner yr un faint o saffrwm, a berwch hwynt am ychydig funudau, a rhoddwch ynddo fêl, ne siwgr candi, a photelwch ef, a chymerwch o hono lonaid cwpan dea nos a bore, a hyn a geidw eich corph mewn perffaith iechyd. I mae genyf un peth eto i'r rhai sydd arferol o ysgrifen!!, cymerwch o sug wermod, a rhoddwch yn eich inc, ac ni all llygod mân na Frengig gnoi byth mo'r papur; y rhai sydd ganddynt lawer o ddillar a'r pryfed yn e u bwyta, rhoddwch dail y wermod yn eu plith, a by 


\section{6}

a'u ceidw rhag pryfed. Ni ddywedaf ddim yan ychwaneg am y wermod, y mae hir ddydd haf yn rhy fychan i ddywedyd yn gyflawn am eu rhinwedd.

\section{White Lilly, neu y Lili wen.}

Maent yn tyfu mewn gerddi; berwch hwynt mewn gwin gwyn, ac yfwch o hono rhag gwenwyn; a chymeryd eu sug yn lle dwfr i dylino torth haidd, a'i chrasu a'i bwyta sydid dda rhag y dropsi; eli o'r gwraidd a'r bloneg sydd yn iachau crach drwg yn y pen, ac yn asio gewyn pan fyddo wedi tori; y gwraidd wedi eu berwi mewn gwin gwyn sydd yn cynnorthwyo i wraig fyned i'w gwely, ac yn dyfod a'r ail i lawr; y gwraidd wedi eu rhostio mewn lludw poeth, ac wedi eu tymheru â bloneg sydd yn blastr anffaeledig i dori chwydd, neu ryw beth dolurus arall; a'r eli a wneir o honynt gyda bloneg a chwyr melỵn, a iachâ losg tân. 


\section{BRITISH HERBAL,}

\section{savo \\ LYSIEULYFR BRYTANAIDD \\ Y ME IDIDID}

YN CYNWYS

AMRYWIOL LYSIAU MEDDYGINIAETHOL,

A dangosiad eglur, pa Blaned sydd yn rheoli pob un o $0^{y}$ amrywiol Lysiau.

Ynghyd a Thabl i ddangos pa Blaned sydd yn rheoli ar bob awr yn y dydd; a thrwy y cyfarwyddyd hwn gellwch dori y llysiau yn yr amser ag y bo y planedau yu rheoli arnynt. Hefyd amrywiol Gynghorion, Eliau a Phlasterau.

Yngbyd ag amrywiol enwau ar. y llysiau.

\section{Wild Carrots, Moron Gavylltion.}

Y maent yn tyfu yn wylltion, ac yn gyffredin trwy y gwledydd hyn ar ochrau y caeau, ar dir gwyllt yn lled gyffredin, ac yn blodeuo ac yn badu yn niwedd yr haf; ac y maent yn ${ }^{-}$dra rhinweddol rhag y grafel a'r gareg, ac yn dda i dori ac i chwalu gwynt, ac i symud pigiadau o'r ystlysau; ac y maent yn dda i ddyfod a'r cyrsiau misol i lawr, ac i wellhau y dropsi, a phob rhyw chwydd yn y bol; ac os bydd eisiau chwalu gwynt; y maent hefyd yn tori y colic, ac yn gostwng, neu yn tawelu gwynt, neu ffitiau y fam; ac y mae eu had yn dda i'w cymeryd mewn gwin; ac hefyd gan y rhai sydd yn anmhlantadwy, os na byddant dros ben eu hamser; yn allanol y mae y dail, ynghyd a mêl, yn gwellhau hên friwiau drewedig a dyfillyd. Mi a allwn benderfynu mai yr hâd yw y rhan oreu o'r llysieuyn 


\section{8}

hwn; gellwch eu herwi mewn dwfr a mêl, ac ychydig win, ac yfwch yn helaeth o hono jn glaear ac wrth eich angen.

\section{Briony, Bloney y ddaear.}

Y inaent yn tyfu ar fryniau, neu diroedd uchel, a than ochrau cloddiau a'u gowraidd yn bur ddyfnion, ac yn blodeuo yn mis Gorpenaf, ac yn Awst; y mae y dail yn rhimweddol rhag doluriau yn y pen, a phen ysgafnder a ffitiau, trwy eu bod yn purgio llawer o humors o'r pen, ac hefyd yn gwellhau y cymalau a'r gewynau, am hyny y maent yn thinweddol thag y polsi, conffylsiwn, a'r cylymau gwythi, ac hefyd yn dda thag y dropsi a'r grafel, trwy agor atalfeydd $\mathrm{y}$ 'splin, a meddalhau ei chaledrwydd; ac y mae eu gwraidd, sedi eu powdro, a'u cymysgu â mêl a'i gymeryd, yn glanbau y frest oddiwrth y phlegm, ac yn iachau hên besychu sydd a diffyg anadl yn ei ganlyn; ac y mae yn gweithio y corph yn bur brysur, am hyny cynghoraf yr angall ei adael yn llonydd; un dram sydd ddigon o'r gureiddyn i'w gymeryd ar unwaith, mewn gwin wrth fyned i'r gwely, un neu ddwy waith yn yr wythnos; gellwch eu defnyddio yn allanol trwy eu berwi mewn dwfr, a golchi y croen âg ef yn brin, wrth fyned i'r gively, rhag y gowahanglwyf, a phob shyw grach dyfillyd, ac anbarddwch ar $y$ croen.

\section{Ash Tree, Onen.}

Berwch y dail ifaingc mewn gwin, ac yfwch $y$ gwin, a rhoddwch $y$ dail yn allanol ar y le a frathwyd gan nadrodd, a phob rhyw ymlusgiaid gwenwynig arall; ychydig o'r dwfe a ddistilir odd:wrthynt 


\section{9}

sydd yn dda i'w "gymeryd bob boreu, gan y rhai sydd dueddol i'r dropsi, ac hefyd i'r rhai sydd yn dueddol i fyned yn rhy dew : y mae y dail, gwedi eu berwi mewn gwin, yn gweithio yn erbyn y grafel a'r clwy melyn; pan nad alloch gael y dail, gwnewch ddefuydd o'r rhisg yn eu lle.

\section{Arsmart, y Benboeth.}

$Y$ maent yn tyfu mewn lleoedd gwlybion, ffosydd ac amrywiol leoedd o'r cyffelyb, y rhai sydd yn sych yn yr haf, yn gyffredin. Y mae sug y dail, a'i rwbio ar chwyydd oer, yn ei wellhau yn fuan;dal ychydig o'r gwreiddyn ar y dant a dỳr y ddannodd; a theflwch $y$ dail ar lawr yr ystafell a hwy a yrant y chwain ymaith yn fuan; ac os rhowch lonaid llaw o'r dail o dan y cyfrwy wrth farchogaeth, fe a drafaelia y march yn llawer gwell; y mae dau fath o'r dail hyn, sef, poetlion ac oerion, ond y poethion yw y rhai hyn, gellwch eu hadnabod wrth eu treio ar eich tafod; an eu bod yu boeth.

\section{Black Hellebore, Crafangc yr Arth.}

$\mathrm{Y}$ maent yn tyfu mewn gerddi. Mae y dail hyn yn lled enbyd i gymeryd llawer o honynt yn dufewnol; and os digwydd $\mathrm{i}$ chwi niweidio eich hunain drwy gymeryd gormod o honynt, y feddyginiaeth yw cymeryd llaeth geifs, os gellir ei gael, onid $\hat{e}$ cymerwch y nesaf i'ch llaw. Mae ei gwraidd yn rhagorol dda yn erbyn pob math o ddoluriau sydd yn tarddu oddiwrth y melancoli, a gwaligofrwydd, y ffitiau, a'r gwahanglwyf, a'r drau glwyf, y melyn a'r du, a'r conffylsiwns, ac y maent yu rhinweddol $i$ ddy fod a'r terms i lawr. Yuallanol y maent yo 
jachau briwiau, trwy roddi y powdr arnynt. 20 grain am ben 10 o cinnamon sydd ddigon i'w gymmeryd ar unwaith.

\section{Polypody af the Oak, Rhedyn y Dderwen.}

$Y$ maent yn tyfu ar amrywiol goed, ond y maent yn cyfrif y rhai sydd yn tyfu ar y dderwen yn well; maent yn wastadol yn leision, gellwch eu casglu pan y byddo angen am danynit. Maent yn dda yn erbyn y cryd, yn enwedig os cymerir mewn gleision maidd, y maent hefyd yn dda i feddalhan y splîn a'r colic, y maent yn ddiniweid, gellir cymeryd dram yn y boreu mewn mêl a dwfr, i weithio y corph yn raddol; mae y dail a'r gwraidd wedi en berwi yn yn dda rhag pesychu a diffyg anadl, gwichian yn $\mathrm{y}$ gwddf; rhaid rhoddi ynddo ddigon o siwgr candi yo helaeth; powdr o'r gwraidd wedi ei gynysgu â mêl, a'i roddi ar gymalau a fyddo wedi eu streifio, sydd yn help mawr iddynt.

\section{Queen of the Meadows, Brenhines y Weirglodd.}

Maent yn tyfu mewn gweirgloddiau gwlybion yn fwyaf cyffredin. Y maent yn blodeuo holl fisoedd yr haf. Eu defnydd mwyaf ydyw stopio pob math o waedu, sef y clwyf gwaed, gwaedu o'r genau a'r ffroenau, cyrsiau arferol a'r gwynion hefyd; fe ddywedir eu bod yn hel ymaith y cryd, a'u bod yn llawenhau y galon; ac fe ddywedir eu bod yn feddyginiaeth anffaeledig i'r colic; y ffordd i'rv cymeryd rhag y doluriau uchod, ydyw ell berwi mewn gwin coch, a'u hyfed. 
Solf-heal, Llysiau'r Cryman.

Y maent i'w cael mewn caeau a lleoedd coediog trwy'r gwledydd. Y maent yn addas i'w berwi megis diod ddail, a' hyfed gan y rhai sydd a briwiau arnynt, pa un bynag ai yn dufewnol ai yn allanol y byddont; ac y maent yn dda i'r rhai sydd wedi briwio oddi mewn, trwy godymau neu gyfryw bethau, ac i'r rhai sydd yn piso neu boeri gwaed o herwyda sigdod tufewnol; yn allanol maent yn dda mewn briwiau hên a newydd, y mae eu sug yn dda i rubio y talcen rhag poen mewn pen, ac y mae eu sug yn dda i olchi y genau a'r gwddf, wedi ei gymysgu ầ mêl.

\section{Ivy, Eiddew.}

Fe ddywedir fod un dram o'r blodeu i'w hyfed ddwy waith yn y dydd mewn gwin coch, yn stopio rhyddni a'r clwy gwaed; ac fe ddywedir fod $y$ grawn melynion yn dda gn erbyn y clwy melyn, ac os cymerwch o honynt cyn yfed cwrw neu licar hwy a'ch cadwant rhag meddwi, ac hefyd rhag poeri gwaed. Nid wyf yn canmol eu cymeryd yn dufewnol, an eu bod yn ddrwg i'r nerves a'r gewynau; y maent yn eu cryfhau, os cymerwch hwynt yn allanol, ac hefyd y mae eu sug yn iachau briwiau a llosg tân; y mae sug y dail, os gwnewch ei snuffio i'r ffroenau, yn purgio'r pen a'r ymenydd, a'r rheum sydd yn cochi y llygadau; eu sug sydd yn dda i'w roddi yn y glust pan bo'n dyfrio; a dywedir, os gwna'r rhai sydd yn cael eu blino gan y splîn, yfed eu diod gyffredin o gwpan wedi ei gwneud o bren leiddew, y byddant lawer iawn gwell; ond shaid i'r iddiod sefyll ychydig yu cwpan cyu ei yfed. 
Service Tree, Pren Criafol.

$Y$ mae ffrwyth $\mathrm{y}$ pren hwn pan y byddont yn addfed dda $i^{\prime} w$ berwi yn ddiod rhag rhyddni a chlwyf y gwaed, a'i gymeryd ynghyd a gwin coch, gellwch eu hyfed yn helaeth; hefyd eu berwi mewn dwfr, a golchi yr aelodau clwyfus âg ef. Nid oes ond ychydig ganmoliaeth i'r dail hyn, ond fe allent fod yn dda er hyny.

\section{Strawberries, Mefus.}

Y mae y dail hyn yn oerion yn eu natur; os bydd eisiau oeri yr jau neu yr ysgyfaint, gellwch ei wneud drwy gymeryd y mefus a'u bwyta, y maent yn oeri y gwaed, yr ysplin, a'r cylla, ac yn tori syched, ac yn atal clwyf gwaed, a'r cyrsiau misol: ac y mae y dwfr a ddistillir o honynt yn dda $\mathrm{i}$ oeri y llygadau ac i wisgo'r bysen.

\section{Acrimony, Llysiau'r Dryw.}

$\mathrm{Y}$ mae y dail hyn yn dda $\mathrm{i}$ wneuthur eli o honynt, i iachau y gout; wrth eu cymeryd yn dufewnol y maent yn iachau briwiau oddi mewn, ac y maent yn iachau y rhai sydd yn gwneud dwfr gwaedlyd. cliriant eu dwfr yn fuan; y maent yn dda rhag y grafel, ac y maent yn un o'r dail goreu rhag y cancer, trwy eu hyfed yn fynych, a'u rhoddi yn allanol ar y lle; ac ni ellwch gael eu gwell i gryfhau yr iau ac i'w glanhau o'i holl ffaeledd.

\section{Barberry, Pren melyn.}

Y rhisg isaf o'r pren, wedi ei ferwi, ac yfed hane peint bob bore oddiarno, sydd yn iacbau y corph 
o's afiechyd, a's anmhurdeb sydd yn y gwaed, sef peudduynod, scurfy, ymgrafu, a phob ryw gyffelyb bethau, ynghyd a'r clwyf melyn; gellwch wneud defnydd o hono i oeri y gwaed a'r iau, ac hefyd y clwyf gwaed; y maent yn ychwanegu yr ystumog at fwyd pan fyddo wedi colli; os rhag y clwyf gwaed, cymerwch y rhisg, a berwch hwynt mewn gwin coch, ac yfwch o hono yn fynych.

\section{Small Centaury, Bustl y dilaear.}

$Y$ mae y dail hyn yn agor atalfeydd yr iau a'r bustl, a'r ysplin; ac yn gwellhau y clwyf melyn a'r dropsi, ac y mae yn sicr eu bod yn lladd y llyngyr yn y bol; mae un dram o'r dail wedi eu powdro mewn gwin yn aml, yn gwellhan brathiadau neidr; ac y mae y sug, os dyferir ef i'r llygaid, yn eu clirio, ac yn cryfhau y golwg.

\section{Adder's tongue, Tafod y neidr.}

Os yfir sug y dail hyu yn fynych, y maent yn feddyginiaeth at bob math o friwiau mewn brest a'r ymasgaroedd, neu ryw barthau tufewnol arall o'r corph; y maent yn cael eu rhodd yn Ilwyraiannus i'r rhai sydd yn dueddol i daflu i fynu o'r cyila, neu waedu o'r genau neu y froenau; y mae cu sug, a'i rymeryd yn y dwir a ddistillir oddiar impiau derw, yn fynych, yn rhagorol dda i'r rhai ag y mae y cyrsiau misol yn dyrod yu rhy helaeth; ac yn allanol gellwch eu defuyddio at bob math o friwiau newydd, trwy eu goìchi â'u sug.

\section{Villow-tree, Helyg.}

$Y$ dail hyn a ddefuyddir i siopio gwaedu, mewn briwiau, gwna :y rhisg neu'r hâd y tro, y maent gn 


\section{4}

stopio cyfogi yn yr un modd, ac y maent yn stopio yr humour hallt sydd yn dropio i lawr ar y sgyfaint. Yr un peth sydd yn unagu consumsions yn aml; ac yn allanol, lludw o'r rhisg, wedi ei gymysgu â finegr, sydd yn tynu defaid oddiar y dwylaw neu ryw fan arall, a'r cyrn oddiar y traed, drwy eu rhoddi ar y lle, y ffordd i'w cymeryd yn dufewnol ydyw eu berwi mewn gwin coch a'u hyfed.

\section{Sinallage.}

Y maent yn tyfu o hosynt eu hunain mewn tiroedd lled wlybion, ond hwy a ffynant yn burion mewn gerddi, os plennir; eu rhinwedd yw agor atalfeydd yr iau a'r ysplîn, ac yn puro'r gwaed; y maent yo helpu rhai sydd yn faelu gwneuthur dwfr; ac fe ddywedir eu bod yn dda rhag y clwyf melyn; $y$ ffordd oreu i'w cymeryd yn dufewnol, ydyw, tynu eu sug a'i ferwi ynghyd â siwgr, a'u hyfed, $i$ iachau y doluriau uchod; yn allanol y mae eu sug, ynghyda honey of roses, a golchi y genau a'r gwddf âg ef, yn fynych yr iachau yn fuan.

\section{Savine, Ffenigi.}

$r$ maent yn tytu yn y gerda, ac yn leision trivy y gauaf; os gwnewch eu sychu a'u powdro, a'u cymysgu â mêl, a'u rhoddi mewn hen friwiau pan y bo eisiau eu glanhau, ac yn methu ei "wneud $\hat{a}$ dim arall; ond fe a'i cadwant rhag croeni; ac $y$ mae yn dda i dori nafod, king's evil, trwy eu rhoddi ar $y$ lle, a'i daenu ar ledr, a'i roddi ar y bogail, y mae yn lladd y llynuyr sydd, yn y bol: ac y maent yn gwellhau y crafu a chrach yn mhob rhan ar $y$ 
corplu; a chwi a ellwch yn llwyddiannus ddefnyddio yr eli ar friwiau sycld yo tarddu oddiar y poz. Hyn am eu rhinwedd yn allanol, ond dim am danynt yn dufewnol, o herwydd eu bod yn thy beryglus i' $*$ cymergd.

\section{Onions, Wynwyn.}

$Y$ maent yn lladd llyngyr ar blant, eu rhoddi yn ngwlych tros nos, ag iddynt yfed peth o'r dwfr yn $y$ boreu cyn tori eu newyn, a'u rhostio yn y lludw poeth, a'u cymysgu â mêl, a'u bwyta, sydd yn tori pesychu, ac yn codi y phleguns i fynu yn fwy essuwyth; yn allatol y maent yn dda, hyny $y w$, ea sug, i wellhau briwiau llosgi neu scaldiad, neu losgi a phowdr; en defnyddio yn nghyd a finegr sydd yn glauhan'r croen oddiwrth spots a phob anharddwch ara!!; dropio eu sug i'r clustiau sydd yn tori y boen a'r swin ynddynt yn fuan.

\section{Ouk, Derwer.}

$Y$ thisg isaf sydd yn cael eu defnyddio yn gyefredin thag poesi gwaed a'r clwyf gwaed; y mae powdr afalau derw, fa'i gymeryd mewu gwin coch, yo atal pob matho waedu ar fab a merch; y maent yo effeithiol i atal y terms ar $y$ inerched.

\section{Mullein, Surcyn y melinyeld.}

T. maent yn tyfu mewn cloddiau ac ochrau y. Siryrdd yu gyffredin; ychydig o'r gwreiddyn wedi ei bowetro, ari gymeryd mewn gwin coch, sydd yn atal rhyddni yn y bol, ac yn ei dawelu; a'u berwi mev'n duff, a'i yfed, sy'n dda i'r rhai tueddol i'r 
cylymau gwythi a'r confylsions, ac ir rhai sydd yn cael eu blino gan hen besychu; golchi y genau âg ef sydd yn dofi y ddannodd; yn allanol, y dail hyn, wedi eu berwi gyda sage a chamomile, a golchi y gewynau $\hat{a}$ hwynt, sy'n eu hiachau; tair wns o'r dwfr a didistillir o honynt, a'i gymeryd hwyr a bore am hir amser, sydd yn iachau y gowt; ac y mae eu gwraidd wedi eu powdro, a'u rhwbio ar ddant pan b'o eisiau ei dynu yn ei wneud yn haws i'w dynu.

\section{Mosses, Mavogl.}

Y mae mwsogl y ddaear yu dda i'w berwi mewn gwin coch, a'i yfed rhag y grafel, ac yn tori a gwasgaru y gareg fel y gellir cael ymadael a hi wrth wheud dwfr; $y$ mwsogl sydd yn tyfu ar dderw sydd yn rhwymo y corff ac yn atal rhyddni a gwaedu yn inhob rhan o'r corff; dyweàir eu bod o'r un natur â'r coed y byddont yu tyfu arnynt; $y$ mae y rhai a helioch oddiar y dderwen, a'u berwi mewn dwfr, a'u rhoddi i'r merched, yn atal y cyrsiau misol pan $y$ byddont yn rhy helaeth; y maent hefyd yn atal y cyfog a'r eigian, ond eu berwi a'u hyfed; ac fe ddywedir eu bod yn cysuro y galon; cymeryd eu powdr yn fynych ar ddiod frag a wellhâ y dropsi.

\section{St. John's wort, Y Gantwll.}

Nid oes en gwell yn tyfu, wedi en berwi a'i wneud yn ddiod, a'i yfed, gan y rhai sydd a briwiau arnynt, pa un bynag ai yn dufewnol ai yn allanol y bo'nt; eu cymeryd yn ddiod sy'n feddyginiaeth hollol i friwiau; eu hadau sydd dda, os yfir oddi arnynt am ddengain niwmod ar ol eu gilydd, rhag $y$ ffitiau a'r polsi. 


\section{House-leek, Llysiau pen lai.}

Y mae posel o'u sug yn dda rhag fitiau poethion o'r cryd, y maent yn oeri ac yn puro y gwaed, ac yn disychedu y sychedig; y mae eu sug yn dda, os diferir ef i lygaid cochion, i'w hoeri; yn allanol y maent yn dda i wneud eli o honyut i wellhau tân eiddaw a llosg tân, ac y mae eu sug yn tynu ymaitli gyrn a defaid oddiar y traed a'r dwylaw; curwch y dail, a rhoddwch hwynt ar goryn y pen, a hwy a ataliaut $\mathrm{y}$ gwaedlyu yn fuan; $\mathrm{y} d \mathrm{~d}$ fr a ddistillir o honynt sy'n dda'ir holl bethau tufewnol a enwyd; os rhwbiwch $\mathrm{y}$ dail ar le wedi ei bigo gan wenyn nell gacwn, fe dderfydd y boen yn fuan.

\section{Hops.}

Gwaith y dail hyn yw agor atalfeydd y 'plin a'r iau, i hel ymaith y grafel, i buro'r gwaed, ac i ryddhau $y$ bol, pan y byddo yn rhwym; ni waeth pa un ai y gwyllt ai y dôf o honynt; y maent yn gwellhau yr ymgrafu, crach; a phob rbyw doriadau allan trwy y corph; ac y maent yn gwellhau y French pox; y maent yndda i'r rhaisy wedi cymeryd grvenwy:- haner dran o'r hadau yn bowdr; a'u cymeryd mewn diod frag in y bore, a ladd y llyngyr.

\section{Hawthorn, Draenen wen.}

Y mae yr had a gewch yn y grawn, ueu y clapiau sy'u tyfu amynt, wedi eu powdro, a'u cymeryd mewn gwin gwyn, yn rhagorol o dda rhag y grafel, y gareg a'r dropsi ; yr hadau, wedi eu glanhau, a'u berwi mewn gwin, a'i yfed, sy'n tori poen oddi mewn; os gwlychwch gadachau poethion yn y dwfr 
* ddistillir o honyut, a'i roddi lle byddo draesz ynddo, ond ei newid amrywiol weithiau, yna cwetwch y ddraenen yn rhoddi meddyginaeth i'w phigiad ei hun.

\section{Groundsel, y Ben felen fanyu.}

Nid oes eisian mo'u gwell at bob math o ddolurinu wedi eu magu trwy wres ac eisiau eu hoeri; os bydd y cylla yn afiach, hwy a godant gyfog, ac os bydd y cylla yn iach, hwy a weithiant $i$ lawr gn raddol; eu sug sydd yn dda i'w cymeryd mewn gwin i wellhan y fitiau a'r clwyf melyn, hefyd is thai sy'n traelu gwneud dwfr; y maent yn dda $\mathrm{i}$ weud powltis i'w roddi ar fronau merched pan $s$ byddont yn chwyddo ac yn cochi; curo y dail yaghyd a halen, a'u rhoddi ar chwarenau, hwy a chwalant; gellwch gymeryd y dwf a ddistillir o honynt yn dufewnol rhag y dolurian an enyd uchod, yn ol eich rheswm.

\section{Golden Rod, y Wialcu euraidd.}

$Y$ maent yn tyfu mewu lle agored, yn mysy coed yn gyffiedin. Y mae y dideilen hon yn cael ei chyfrif yn anghydmarol rhag y gareg a's gratel, ac i wneud dwf newn helaethrwyd; dywedir ei bod yn atal gwaed o'r holl ranau tufewnol; $y$ mae yn rhagorol o dda mewn diod ddail, i wellhan briwiau yn allanol ac yn dufewnol; ac os berwch $y$ dail hyn gyda sage, a thoddi ynddo ychydig alwm, a golchi eich genau a'ch dannedd âg ef pan y byditont, yn rhydd, a hyny a'u gwnant yn sound yn fuan.

\section{Garlick, Garlleg.}

Y maent yn dda rhag brathiad ci drwg, a plob 
Thyw ymlusgiaid gwenwynig ereill; ac y maent y lladd llyogyr mewn plant; ac y maent yn dda i dori, $i$ godi, ac i daflu y flems mwyaf gwydn; ac y maent yn dda os bydd un gwedi ffieiddio ei hunan wrth yfed dwfr drwg, neu fwyta, dail gwenwynig, megis cegyd, neu'r cyfryw bethau peryglus o un rhyw. Defuyddiwch hwynt yn helaeth yn allanol; ond yn dufewnol cymerwch hwynt yn lled brin. Nid yw yn ganmoladwy i'r rhai sydd a natur melancoln arnyut.

\section{Fumitory, Musogl y ddaear.}

Y mae y dail hyn yn dda i agor atalfeydd, yr iau, a's ysplin, ac yn puro $y$ gwaed, ac hefyd yn gwell-. hau y clwyf melyn; ac os cymerir y powdr o'r dait sychion, am hir amser, y maent yn gwellhau y melancoli. Eu sug, trwy ei ddiferu i'r llygaid, sydd yn eu gwellhau pan ag y b'ont yn gochion ag eisiau eu hoeri. Sug y dail hyn a dail tafol, a'r un faint o vinegr, a golchi y lle âg ef, sydd yn iachau polo math o grach.

\section{Elicampane, March Alarch.}

Y mae y dail hyn yn iachau ffaeledd y frest, os Q oerfel y magodd, ac y maent yu tori y cnoi yn y rest, a'r pigiadau sydd yn yr ystlysau, y rhai sydd n codi oddiar yr ysblin; ac y maent yn gwellhau pesychu, diffyg anadl, a gwichian yn y frest; y naent yu atal cerddediad brathiadau nadrodd. $Y$ aae y gwraidd a'r dail gwedi eu curo, a'u rhoddi ingwlych mewn cwrw, ac yfed o hono bob boret, on glanhau, ac yn cryfhau, ac yn sirioli y llygaid yn hagorol; a'r dail, gwedi eu berwi mewn gwin, a'u 
hyfed, sydd yn hel ymaith bob math o longyr o's corff; ac y maent yn dda i'r rhai sydd yn poeri gwaed; y gwreiddyn wedi ei guro a'i ferwi mewn vinegr, a'i wneuthur yn eli, ynghyd a saim traed defaid, sydd yn gwellau yr ymgrafu ar hên ac ieuangc; ac os berwir y dail a'r gwraidd mewn dwfir a vinegr, a golchi y lle âg ef, etyb yr un dyben, ac y mae yn beth parotach.

\section{Dove's foot, y Goes goch.}

$Y$ mae y dail hyn gwedi ell profi yn dda i dor y gwynt a'r poenus ofidiau sy'n berthynol iddo: a i dori y gareg, ac i chwalu y grafel. Y mae hi y rhagorol o dda; hyn a brofais yn aml fy hun. mae llawer yn eu cnocio a'u rhoddi ar friwiau ne: wydd, a hyn a'u gwellhâ yn fuan; y mae y dail y dda, wedi en berwi a'u hyfed yn barhaus gan y rha sydd wedi tori eu llengig; nid yn aml y cewch " givell at $y$ dolur hwn.

\section{Daisies, Llygaid y dydd.}

$Y$ mae y rhai mawr o honynt yn ddail briwiz da, yn dda i'w gwneur yu eli ac i'w hyfed ynoddic at bob math o friwian; a'r rhai bychain, sydd $y$ tyfu yn y caeau, sydd yu bur dra yn noluriau frest, pan b'o arni eisiau ei hoeri; yn allanol y dz yu bowltis, sydd yn meddalhan chwydd coch chaled; a'i sug sydd yo dcla i'w ddiferu i's llyga clwyfus; ac y mae y dail, grwedi eu cnocio a'u rh ar y lle, yn chwalu chwarenod, yn mha le bynag: byddont, ac yn llwyddianus y gwneir eli o hony at friwiau. 


\section{1}

Crow-food, Crafangc y frán.

Nid yw y dail hyn i'w cyneryd yn dufewnol, ond i'w definyddio yn allanol, a'u cymysgu â mwstard, a hwy a blasdrant, yn gystal a dim a ellwch gael, ac yn fwy diberygl.

\section{Costmary, Tafod yr oen.}

Y mae y ddeilen hon yn gyffredin, ac yn bawdd ei chael, ac yn rhagorol o dda rhag y grafel, ac yn meddalhau caledrwydd yr ysplin, ac yn cryfhau yr iau a'r holl ranau tufewnol, ac yn dda rhag ffitiau o'r cryd; y mae eu haduu yn cael eu rhoi, yn llwyddiannus, i blant yn erbyn y llyngyr, y mae y dail hyn a thafod y neidr, ac olew, yn gwneud eli da at friwiau.

\section{Hemlock, Cegyd.}

Yr wyf yn eich rhybuddio yn flaenorol nâ wnewch dim defnydd o hon yu dufewnol; ond yn allanol, y maent yn dda mewn powltis $i$ ostwng hhwydd, ac yn ddigon diberygl ar bob than o'r rorph, ond yn unig ar y dirgeloedd, cadwch hi oddi wo: ond os bydd angenrheidrwydd yn galw, sef y pox, peidinch a i dial ond lleied ag y galloch; i ynu y bysen oddi ar y llygad, cymerwch ychydig 'r dail hyn, a'r un faint o bay salt, a'u cnócio yn gliyd, a'u rhoi wrth arddwrn gwrthwyneb i'r llygld dolur, a'i wisgo a'i newid yn awr ac yn y man, hyn a dyna y bysen, a'i newid bob 24 o oriau: s bwyty. neb y dail mewn camgymeriad yn lle arsly, y feddyginiaeth yw yfed y gwin goreu, yn elaeth. 


\section{3}

Prony, Blodau y brenin, nex Rosyn y mynydd.

Y mae y gwraidd hyn yn annghydmarol o dda atal cyfog o bob math, yn fuan, y tro cyntaf neu yr mil; a chymerer y powdr o'r gwraidd mewn rhyw weth cyfaddas; ac y maent yn iachau y fitiau, os cymerir hivgut mewn pryd.

Fy amcan yu bresenol yw dangos pa blaneo sydd yn rheoli pob un o'r llysiau a grybwyllir an darynt yn y llyfr bw' fel y gallo y darllenydd trwy y cyfarwyddyd a roddir yma, adnabod o dat ba blaned y mae pob liysieuyn yn tyfu, fel y gallocl ddyfod a'r feddyginiaeth yn agos at y clwyf; o bydd eich dolur yn gyndyn i wellhau, cesglwch : dail sydd yn gyfaddas, wrth y cyfarwyddyd yma torwch y dail pan b'o y planedau yn rheoli arnyn fel y canlyn; mi a enwaf y dail a'r planedau syd yn rheoli arnynt yu gyferbynio â hwyt yu $z$ dul canlynol:- -

Arach-wild, Venus Adder's tongue, Moon Acrimony, Jupiter

Angelica, Sun Ash-tree, Sun Arsmart, Mars Balm, Jupiter Barberry, Mars Bay-tree, Sun Betony, Jupiter Blue-bottles Saturn Briony, Mars Burdock, Verus
Bugles, Venus Bare-foot, Mars Broom, Mars

Buck's-horn Saturn Camomile, Sun Celandine, Sun Comfrey, Saturn Carrots, Mercury Colt's-foot, Venus Crow-foot, Mars Dandelion, Jupiter Dwarf-elder, Venus Dove's-foot, Moon 
Daisies, Venus

Eringo, Venus

Eye-bright, Sun

Elicampane, Mercury

Fern, Mercury

Water-fern, Saturn

Feunel, Mercury

Fox-glove, $V \in$ nus

Fumitory, Saturn

Golden-rod, T'enus

Gooseberry-bush, Venus Red-roses, Jupiter

Ground-ivy, Venus

Hemlock, Saturn

Helebore, Saturn

Hysop, Jupiter

Hops, Mars

House-leek, Jupiter

Honey-suckle, Mercury

Honey-thistle, Mars

Hawthorn, Mars

St. John's-wort, Sun

Ivy, Saturn

White-lillies, Moon

Maiden-hair, Mercury

Mallows, $V$ enus

Marygold, Sun

Mint, Venus

Mother-wort, Venus

Mouse-ear, Moon

Mug-wort, Venus

Mulberry, Mercury

Mullein, Saturn

Mustard, Mars

closses, Saturn
Nettles, Mars

Oak, Jupiter

Onions, Mars

Parsley, Mars

Peony, Sun

Pelitory of the wall, Mer.

Pill-wort, Mars

Polypody, Saturn

Queen of the meadows,

Venus

Rosemary, Mars

Garden-rue, Sun

Sage, Jupiter

Sage-wild, Venus

Solomon's-seal, Saturn

Sanicle, Venus

Savine, Mars

Scurvy-grass, Jupiter

Self-heal, Venus

Service-tree, Saturn

Smallage, Mercury

Sorrel, Venus

Wood-sorrel, Venus

Strawberries, Venus

Shepherd's-purse, Sat.

Tansy, Venus

Wild-tansy, Venus

Thyme, Venus

Tormentile, Sun

Vervain, Venus

Willow-tree, Moon

Wormwood, Mars 


\section{4}

TABL O ORIAU Y PLANEDAU, Am bob dydd yn yr Wythnos.

\begin{tabular}{|c|c|c|c|c|c|c|c|c|c|c|c|c|c|}
\hline ul & & Llu & & $\mathrm{M}_{\mathrm{z}}$ & & VI & & & & & & S & \\
\hline $\mathbf{P} \mathbf{l}_{\mathbf{p}}$ & 0. & Pl. & & $P l$. & & $P l$. & 0. & $P l$. & 0 & $P l$. & & $P l$. & $o$ \\
\hline & & 2 & & $\delta^{8}$ & 1 & & & ఫ & & & & & \\
\hline & & & & & 2 & & 2 & & 2 & & & 4 & \\
\hline & & & & & & & & & 3 & & & & \\
\hline & & & & & & & & & 4 & & & (๕) & \\
\hline & 5 & & & & & & 5 & & 5 & & & 우 & \\
\hline & 6 & & & & & & 6 & & 6 & & 6 & $\not$ & \\
\hline & 7 & & & 4 & 7 & 오 & & & 7 & & 7 & ) & \\
\hline & 8 & $D$ & & مิ & 8 & & 8 & 24 & 8 & & 8 & h & \\
\hline & & & & & 9 & & 9 & & 9 & & 9 & & \\
\hline & 10 & 24 & & 8 & 10 & & & & 10 & & & & \\
\hline & 1 & & & & 1. & & 11 & & & & 11 & & \\
\hline & 12 & & & $D$ & & $\delta$ & 12 & & 2 & & & * & \\
\hline & 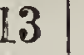 & Q 1 & & h & 13 & 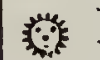 & 13 & $\bar{D}$ & 13 & & 13 & ఫ & \\
\hline & 14 & & & 21 & & & 14 & & & & 14 & 2 & \\
\hline & 15 & & & $\delta$ & & & 15 & & 15 & & 15 & & \\
\hline & & & & & & & 26 & & & & 16 & & \\
\hline & 17 & 2 & & 9 & & h & 17 & & & & 17 & & \\
\hline & 18 & & & & & & 1 & 우 & 18 & & 18 & & \\
\hline ? & 19 & & & D & & $\delta$ & 19 & $q$ & 19 & & 19 & * & \\
\hline & 20 & 우 & & & & (3) & 20 & $D$ & & & 20 & & \\
\hline & 21 & & 21 & 4 & 21 & & & & & & 21 & 2 & \\
\hline 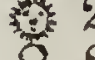 & 28 & & & & & & & & & & 22 & & \\
\hline & 24 & & & & & & & & & & & & \\
\hline & & & & & & & & & & & & & \\
\hline
\end{tabular}

Y tabl sydd mor hawd fel mad oes eisian on schydig eglurhad arno. Wi diefnydd ydyiv, ca pa blaned, sydd yn theoli unrhy awr yn y dydl bob dydd o'r wythnos. Gan roddi esianpl, yr y wyf yn deisyf gwybod pa blaned sydd yn rheoli dý Mercher, saith o'r gloch y nos; o dan yr enw'dye Mercher, edrycher am 19, yi hyn sy'n ateb i sai 


\section{5}

o:r gloch y nos, canys y dydd naturiol sydd yu cyn nwys 24 awr, yn dechreu ar ol haner nos, felly y mae oddiwrth 12 ganol dydd, a dechreuwch gyfrif $13,14,15$, \&c., a chwi a gewch weled inai y 19 awr o haner nos sydd yn ateb i saith o'r gloch y nos, a chyferbyn â hyn chwi a gewch ơ yr hyn. sydd yn dangos hon $i$ fod yn awr i Mars. Ac os mynwch wybod pa blaned sydd yn rheoli ar saith yn y bore y dydd hwnw, chwi a gewch gyferbyn ? 7 , yr hyn sydd yn dangos mai Venus sydd yn rheoli yr awr hono; ac felly am unrhyw awr arall yn y dydd.

Wrth y rheol a ddangoswyd yn y tabl hwn, gellwch dori pob deilen yn yr awr ag y byddo ei phlaned ei hun yn rheoli arni, fel ag y gwelwch fod $y$ Sun, sef yr Haul, yn rheoli ar yr awr gyrtaf ddydd Sul, ac felly yn y blaen; gwelwch fod y planedau yn newid bob awr, am y 24: ac felly geill pub dyn sydd yn meddiannu deall naturiol ddeall y tabl hwn fel ag $y$ rhag-ddywedwyd fod $y$

Sun $\odot$ yn rheoli ar ddydd Sul,

Ar ddydd Llun y D

Ar ddydd Mawrth, Mars o

Ar ddydd Mercher, Mercury $\varnothing$

Ar ddy dd Iau, Jupiter 4

Ar ddydd Gwener, Venus o neu *

Ar ddydd Sadwrn, Saturn $h$

Dyma i chwi ddangosiad o'r planedau sydd yn. rheoli yr awr gyntaf am bob dydd yn yr wythnos, a gwelwch wrth y tabl, mai yr un reol sydd am y pedair awr ar hugain, ie am yr wythnos, ond eu bod yn newid bob awr.

Ond rhag eich gwangaloni, gellwch benderfynu fod yr holl lysiau a enwyd yn y llyfr hwn, yn 


\section{6}

gyfaddas feddyginiaeth i'r amrywiol ddoluriau a enwyd yn y llyfr hwn, yn dufewnol ac yn allanol, pa amser bynag y torir hwy,-ond eu bod yn llawer mwy rhinweddol at feddyginiaeth y clwyf yn dufewnol ac yn allanol, os torir hwynt ar yr awr ag y byddo y Blaned yn rheoli arnynt.

$\boldsymbol{Y}$ ffordd i wneuthur Powltis at gasglu yr afiechyd i'r un man, fel ag y gellir ei dori a'i sugno allan.

Had llin, had fenigreek, a'u malu, a'u berwi ynghyd, a'u tewychu â blawd brag, a rhoddi ynddo saim gwyn, neu sweet oil.

\section{Un arall i'r un dyben.}

Cymerwch ddail lili gwedi eu shostio, a'u curo yngliyd â bloneg.

Un arall.

Cymerwch ddail neu wraidd rhocos, wedi eu berwi a'u tewychu $\hat{a}$ blawd brag, a rhoddwch ynddo saim i'w ystwytho.

\section{I dori chwydd coch yn fuan.}

Cymerwch ddail bysedd cochion, a dail duon da, a dail pen felen, a'u curo ynghyd, a'u berwi a'u tew'ychu â blawd ceirch, a'i ystwytho â sweet oil, a'i roi ar y dolur, gan ei newid bob dydd.

$\boldsymbol{Y}$.ffordd i wneuthur Eli da $i$ lanhau hen friwiau drewedig.

Cymerwch verdigras wedi ei bowdro, haner wns, mêl, chwech wns, vinegr, tair wns, a berwch yu- 
ghyd hyd nes y tewycho, a throi yn lliw coch; a defnyddiwch ef mewn hen friwiau i'w glanhau.

Cyfarwyddyd $i$ ddefnyddio amrywiol Oilion $i$ esmuythhau poen.

Oil of sweet almond6, linseed oil, oil of St. John's wort, oil of earth-worms, oil of elder, oil of turpentine, oil of amber, oil of swallow's, oil of

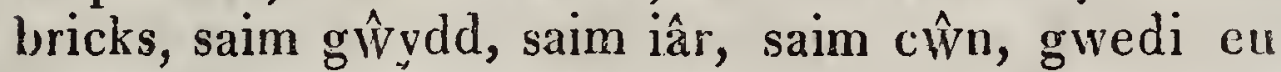
berwi, a hel y saim, camomile, ffenigreek seeds, a dail cwrw. Gan fod yr oilion a enwyd yn adnabyddus i'r wlad yn gyffredin, y maent i'w cael gan y rhai sydd yn goverthu drugs, wrth yr enw a roddwyd arnynt yna; ac os bydd rhyw ran o'r aelodau yn boenus trwy oerfel, cymerwch ryw un o'r rhai a enwyd, neu cymysgwch anrywiol o honynt yn nghyd, a defnyddiwch oflaen y tâu, a lapiwch $\hat{a}$ gwlanen gynes; ond na ddefnyddiwch y pethau at enwyd uchod, os bydd y dolur yn wresog yll ei natur.

\section{I stopio chấydd mewn bron.}

Cymerwch faip gwedi eu rhostio a chnociuch, a rhoddwch ynddo oil rhosus, a newidiwch bob dydd, a golchwch y fron bob tro y newidiwch y pow!tis $\hat{a}$ chamomile a dail rhoccos wedi eu berwi yn nghyd, mor boeth ag y gellir ei oddef, hyn sydd yn dda $i$ chwalu chwarenau.

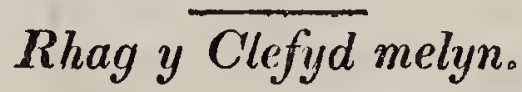

Cymerwch ddwy bilsen yn y nos, o casteel soap a saffrwn, hyd nes y byddoch yn iach, a chadwch oddiwrth ddiod frâg a llaeth wrth ei gymeryd, ac jach a fyddwch. 
Tstopio gwaed mewn briwian.

Cymerwch ddail poethion, a chnociw ch, a ihoddwch ar $y$ lle; neu cymerwch bolarmoniac a thaflwch i'r briw; new cymerwch dragon's blood, a rhoddwch yn y briw; neu cymerwch rosemary, a dodwch yn y dolur, neu blisg wyau, wedi eugwnend yn llwch; neu litharge, neu frankinsense, mastic, neu gobweb, hyny yw, gwe y pryf copyn. Y mae y porrdr a enwyd i'w gael yn y siop, rhyw un o honynt a wna $y$ tro, ond ei falu, a'i roi yn y dolur.

\section{Un arall ìr andyben.}

Cymerwch frankinsense un dram, aloes hanex: dram, wedi eu malu, cymysgwch yn nghyd, a phan y bo angen am dano, cymerwch wnionyn a churwch ef yn dda, yna cymysgwch y pethau a enwyd uchod at dewder mêl, a gwlychwch gudyn o flew ysgyfarnor ynddo, a rhoddwch yn y briw, a gadewch ef yno hyd nes y mendio, ac fe a ollynga ei afael ei hun; mae hyl o gyngor yn fwy ei werth na phris y llyfr hwn i gyd, gellwch ei wneud i fynu â gyerth dwy geiniog yn y siop.

\section{Cyngor i lanhan briwiau oddiwrth gig marw.}

Mi a enwaf amrywiol o'r pethau goreu i'r pwrpas hwn, ac yna gellwch gymeryd eich dewis o honynt, canstic, verdigrease, copperas, vitriol, hyny yw, y gareg las, alum wedi ei losgi, antimony, mercury, sublimate, precipitate, cymerwch eich dewis un o bongut at" yr achos hwu. 
Rhag y Cryd cymaiau pooth, pan ary y byddoch yn methu goriuedd yn y gwely.

Cymerwch hemlock, sef y cegyd mân sydd yn tyfu hyd y cloddiau, a lettice, sydd yn tyfu yn y gerddi, a berwch yn ughyd mewn dwfr, a golchwch y lle poenus âg ef yn fynych, wrth fyn'd i'ch gively. Ac os dewiswch yn well, gellwch chwanegu atynt ddail paladr y wal, a dail rhosus cochion, a'u gwiend yn bowltis a'i roi ar y lle, gan ei newid bob dydd.

\section{Basilicon, Eli i lanhau briwiau.}

$Y$ ffordd i wneud yr eli. Cymerwch gîyr melyn ac ystor, a Greek pitch, o bob un, pedair wns, ac o oil cyffredin, pedair wns a hanes, a'u cymysgu yn nghyd trwy eu toddi yn raddol. Y mae yr eli hwn yn glanhau pob math o friwiau, ac yn eu hesmwythau.

Eli at friwian a thyllau drewedig yn y cocsau.

Cymerwch gum elemy, turpentine, o bob un was a haner, gwêr dafad, ddwy wns, a bloneg mochyn, un wns, a'u toddi yn raddol ar dân arat. Y mae hwn yn eli da rhagorol, ond na ddefnyddiwch mewn un matb o friwiau os byddant ar y gew ynau.

\section{Eli i groeni briwiau:}

Cymerwch oil of roses, chwarter pwys, chyr gwyn, tair wns, bolarmoniac, haner wns, litharge of gold, un wus, camphor, haner dram, yr hyn sydd i'w toddi a'u cymysgu, a'u gwouthur yn eli, a'i gymeryd i groeni ac i sychu briwian. 


\section{Plastr i gryfhau ystumog.}

Cymerwch mastich, un wns, bolarmoniac, chwe' dram, dail rhoses cochion, chwarter wns, cẁyr melyn un wns, oil un wns, a'i wneud yn blastr, a"i roddi ar y frest, wedi ei daenu ar ledr.

\section{Un arall i'r un dyben.}

Cymerwch fintys a wermod, a dail cwrw, o bob un ddram, dail rhoses cochion, dau ddram, a phupur hirion, un dram, mustick tri dram, ystor a chŵyyr melyn, o bob un pedair wns, oil of spice, un dram, oil cyffredin, cymaint a'i gwna yn blastr.

Gan fod amrywiol enwau ar y llysiau, yn adnabyddus yn un wlad wrth un enw, ac mewn gwlad arall wrth enw arall, i'ch gwneud yn fwy adnabyddus o honynt, mi a enwaf yr enw Saesoneg yn gyntaf, ac amrywiol enwau Cymraeg ar eu hol, fel y canlyn:-

Arach-wild, troed yr ẁydd (Briony, hloneg y ddaear, $y$ A crimony, llysian'r dryw, troed $\mathrm{y}$ dryw

Adder's tongue, ta fod y neidr, dail pen y neidr.

Angelica, llysiau'r ysgyfaint, llysiau'r angel

Ash-tiee, onen

Arsmart, y beu boeth

Balm, bồn

Barberry, pren meiyn

Bay-tree, y dail civrw, pren $y$ gelwin

Blue-hottles, pen galed, pen. las yr yd winwydden wen

Burdock, cedor y wrach

Bugles, llysian tafod yr oen.

Bale-foot, chwys Mail

Broom, banadl

Buck's-liorn, llwyn hidì, dail ceiliogod

Camomile, camamile

Celandine, llym y llygaid, llys. iau'r wenol

Comfrey, llysian y cwlwm

Carrots-wild, moron gwylltion

Crow-foot, clafange y fran, chwys Mair. 
Dandelion, daint y llew, daint y ci

Dwarf-elder, ysgaw Mai

Dove's-foot, y droed goch, troed y golomen

Daisies, llygaid y dydd, yr asgen

Eringo, môr gelyn, celyn y môr

Eye-bright, y dorfágl, effros, llygaid siriol, llygaid Crist

Elicam pane, marchalarch

Fern, rliedyn, rhedyn y dufr, rhedyn gy fyrdwyt

Fennel, ffanigl, ffunel

Fox.glove, menyg y llwynog, dail bysedd cochion, dail crach

Fumitory, mwsog y ddaear

Golden-rod, y wialen auraidd

Ground-ivy, eiduaw y ddaear, dail Robin, dail eidrol, llysiau'r esgyrn

Groundsel, y ben felen fanyw

Hemlock, math o gegid mân yn tyfu hyd ochrau y cloddiau, cegid gwynion

Helebore, tafol y môr, troed yr arth, crafange yr arth

Hysop, dail cyffredin yn y gerddi

Hops, yr liwn a roddir mewn cwrw

Horebound, roround, y maent i'w cael yn y gerddi wrth bob un o'r ddau enw

Houseleek, llysiau pen tai

Honey-suckles, dail gwinwydd, lili y dyffryn

Honey-thistle, ygall bendigaid, cribau. Mair

Hawthorn, y ddraenen wen

St.Jolın's-wort, y gantwll, nail Ivy, eiddaw

White-lillies, lili'r gesddi

Maiden-hair, gwallt y forwyn Mallows, dail y rhocos, hocys
Marygold, gold Mair

Mint, mintys y gerddi

Mother-wort, Llysiau'r fam

Mouse-ear, clust y llygoden

Mug.wort, llysiau Ifan, llys. iaullwydion

Mulberry

Mullein, surcyn y melinydd

Mustard, mwstard

Mosses, mwsogl, migwyn

Nettles, dail poethion, danad! poethion

Oak, derwen

Onions, winwyns

Parsley, persli

Peony, blodau y brenin

Pelitory of the wall, paladr $y$ wàl, paradlys

Pile-wort, dail peils, llygaid Ebrill.

Polypody of the oak, math o redyn sydd yn tyfu ar dderw

Queen of the neadow, brenhines y weirglodd

Red-roses, rhosus cochion

Rosemary, rhos Mair

Rue, rbyw y gerddi

Sage, saeds, y fedwen cliwerw Sage-wild, saeds gwylltion, chwerwlys yr eithin

Solomon's-seal, y maent i' $w$ cael yn y gerddi yn gyffredin

Sanicle, clust yr arth

Strawberries, mefus

Scurvy-grass, dail ysgyra

Shepherd's purse,pwrs y bugail

Sorrel, surans y cwn

Woood-sorrel, surens y gôg, sureris tair dalen

Tansy, y maent i'w cael yu gyffredin yn y gerddi wrth yr euw hwn

Savine, ffenigl

Smallage, clo cylor, cnau. ddaear 


\section{2}

Selfheal'

Service-tree, pren criafol

Wild-tansy, y dansi wyllt, y dinlwyd

"Thyme, teim y gerddi

Wild-thyme, teim gwylltion

Tormentile, treiagl y moch, dail gwylltion sydd yn tyfir ar ochr pob clawdd o'r bron Vervain, llysiau yr hudol

Willow-tree, helyg y gwinllanoedd

Womwood, ehwerwlys

\section{CYNWYSIAD Y RHAN GYNTAF.}

Rhag y Darfodedigaeth

Rhag y cryd

I gryfhau'r Ystumog

Rhag brathiad ci cynddeiriog, a nadrodd ....

Rliag poeri gwaed

Rhag piso gwaed

Rhag y Pâs ar blant

Rhag y Consumption mewn hen ac ieuriugc ....

Rhag Ffitian convulsions

Rliag y Peswch

Rhag Llosg tân

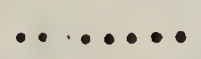

$Y$ ffordd i wnend dwfr llygaid

I attal gwaed mewn briwiau

Rhag dolnr mewn céz..

Rhag y Dropsy ......

I attal y Terms, \&c...

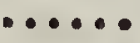

$\ldots$

Cyngor i blant wedi tori en llengig

Rliag y Cauger

I gryfiau y golwg

Rliag ysgafnder mewn pen

Rhag y Colic

Rhag Dolur mewn pen

Rhag $\mathrm{i}$ blant biso yn el gwelyau

Rhag y Elyngy! .....

Rhag y Crygni......

Diod i iachan briwiau ....

Rhag y Clwyf melyn ....

Rhag piso gwaed

Rhag y Ddannodd

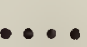

- $\bullet$

$\circ \bullet$

$\ldots .$.

$\ldots$
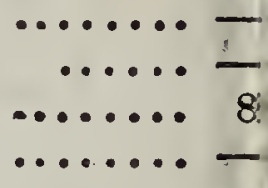

$\cdots$
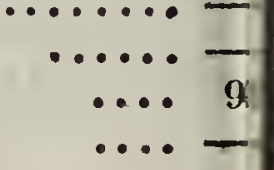


\section{3}

Rhag Dolur y Gareg, \&c. _..... .... .... 18 Cyngor i wragedd rhag Ymysgario $\quad \ldots . . \quad \ldots \ldots . . .19$ Rhag dolur mewn bol ..... Rhag y Polsy Rhag y Piles Rhag y Melancoli I gryfhau y côf
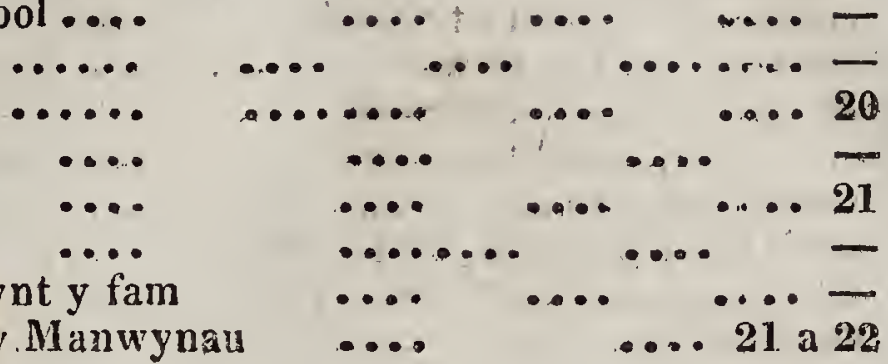
I chwanegu llaeth $\quad \ldots$... Rhag ffitiau neu wynt y fam Cyngor i wellhau y. Manwynau Rhag yr Igian I wellhau y Pox ..... Cyngor i rai sydd a'n gwynt yn drewi ... Cyngor i wellhan y Frech goch Rhag y Frech wea Cyngori rai trwm eu clywed $\ldots \ldots \cdots \cdots=$ I iachau y bysen mewn llygad I wellhau $y$. Parlys a chryndod dwylaw. Rhag y Gout I ehwalu gwynt o'r cylla .... Eli da i lanhau briwiau

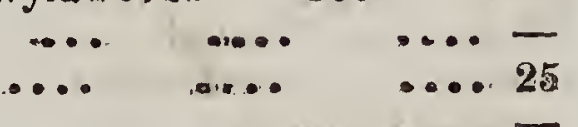

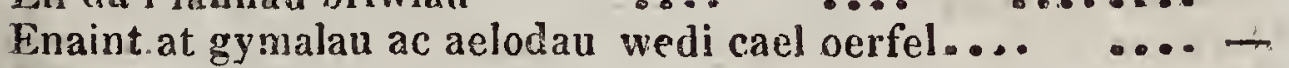
Enaint i'r Nerves ..... ..... .... .... 26 Plastr i dori y Colic .... .... ... ...... Plastr i'w roddi ar esgyrn briwedig, \&c. $\quad \ldots . . \ldots 27$ Eli da at bob math ofriwian Rliag y Scurvy yn y dannedd

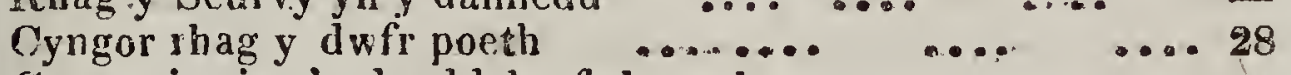
Cyngor i rai sy'n dueddol o forl yn rhwym $\quad \ldots . . . .$. I ostwng chwydd yn mronau merched, \&z. .... .... Cyfarwyddyd i fanaeth brin o laeth.... .... .... Cyngor i wellhau pen y fron $\quad \ldots \ldots \ldots \ldots . . . . . \quad 29$ I dori y Colic ar wragedd, \&c. $\quad \ldots . \ldots \ldots \ldots \ldots$ Rhag cnofa yn molian plant $\ldots \ldots \ldots$ I glyfhau babanod gweiniaid $\quad \ldots \ldots \ldots \ldots \ldots$.... Thag fitiau ar fabanod newydd eni.... ... .... -

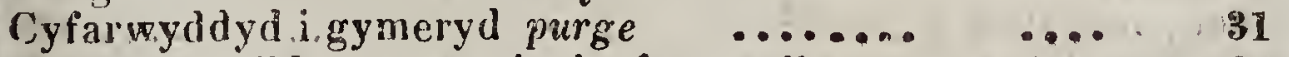
Dull Arac wild, veu .Lysian'r fam wyll .... .... 32

\section{CYNWYSIAD YR AIL RAN.}

Cyfarwyddiadan i gasglı; sychu, ac i gadw dail yn ddilwgr $3 \frac{1}{4}$ Am y Blodan, yr Hadan, a'r Gwraidd.. .... .... 35

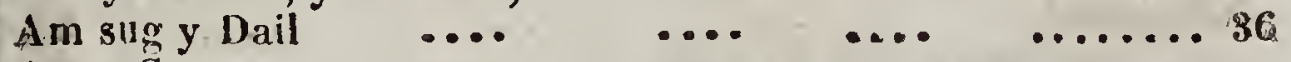
Am y Syrups
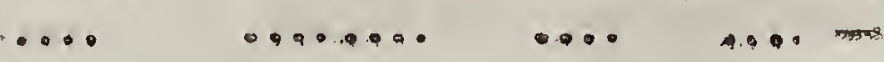
Angelica, neu Lysiau yr Angel.

Balm, ueu Bôm

Betony, neu griban'St. Ffraid

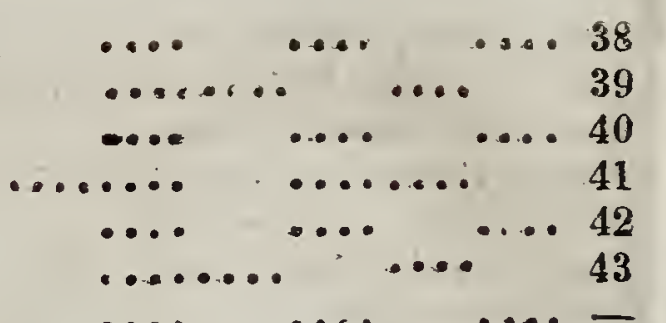

Burdock, nen Cacamwci

Bey leaves, neu Ddail cwrw

Blue bottle, nen y Bengaled

Broom, neu Fanadl

Buck's holn plantain, neu Lwynhidl ...... .... 44

Colt's foot, neu ddail carn yr ebol

Celandine, neu lym y llygaid

Cwmphri

Camomile

Dwarf Elder, neu Y'sgaw Mair

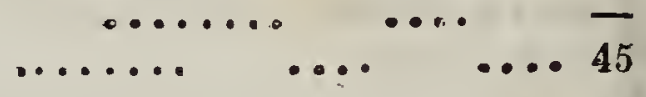

Dandelion, neu Ddant y llew

Eringo, neu Gelyn y môr

Eye-bright, y Dorfagl neu Lygad siriol.....

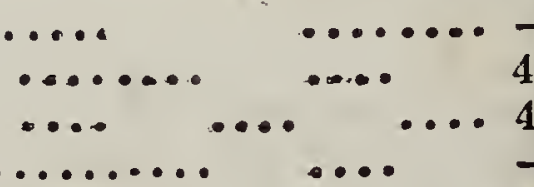

Fox's gloves, neu Ddail bysedd cochion....

Fern, Rhedyn

Fennel, neu lifanigl.....

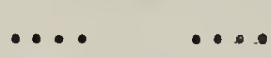

Ground Ivy, neu Eiddew y ddaear

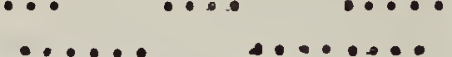

Gooseberry-bush

Horound

Hyssop

Holy thistle, nen Ysgall bendigaid

Honey suckle, neu Ddail gwinwydd

Mother-wort neu Lysiau'r fam

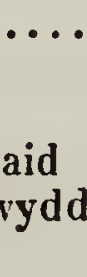

Maiden-hair, neu Wallt y forwyn

Marsh-mallows, Hoccys, neu ddail rhocos

Mustard

Mouse-ear, nei glust y llygoden

Mng-wort, nen Lysiau Evan

Marygold neu Gold Mair..

Nettle, neu Ddanadl poethion....

Pellitory of the wall, Palads $y$ wàl

Pile-wort, Llygad Ebrill

Rhosmari

Rheu, neu Ryw y gerddi

Red rose, Rhosus cochion

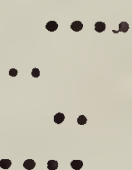

Sage, neul Saeds

Sage, nen Saeds gwylltion ..

Wood-sorrel, neu Surens y gog

Sorrel, neu Surens y cînn ..

Sanicle

Solomon seal...

...

Seurvy-grass, neu ddail Ysgyrff

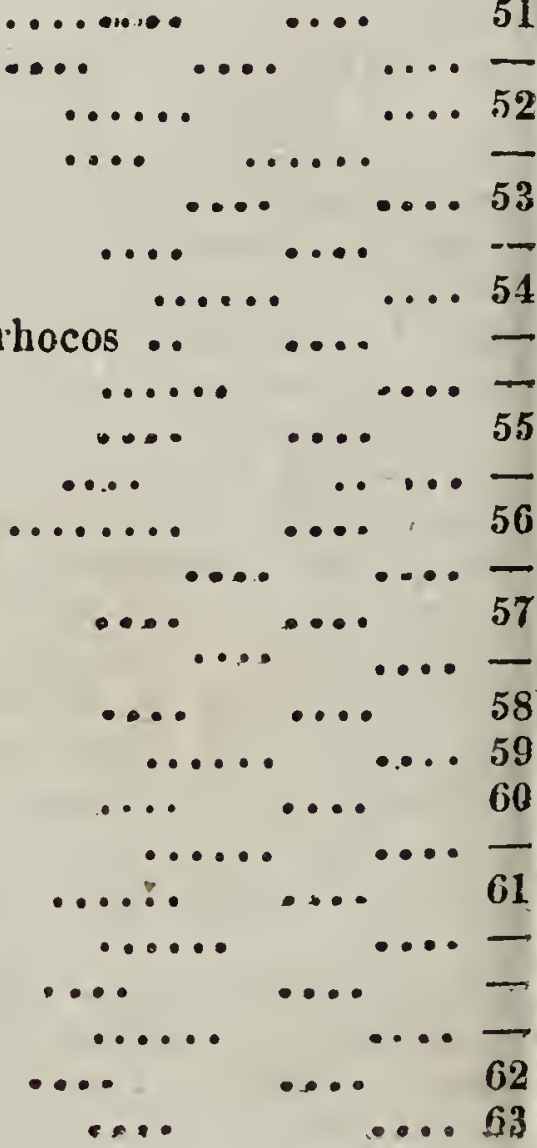




\section{5}

Shepirerd's purse, neu Bwrs y bugail Thyme, Teim

Tormentile, Triagl y moch

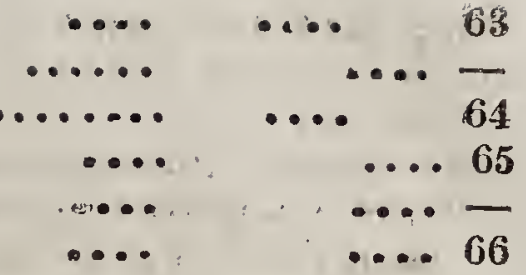

Vervain, neu Lysiau yr hudol..

Wormwood, Wermod

White Lilly, neu Lili wen....

\section{CYNWYSIAD Y DRYDEDD RAN.}

Wild Carrots, Moron gwyllition Briony, Bloneg y ddaear .. Ash tree, Onen Arsmart, y Benboeth ...... Black Helebore, Crafangc yr Arth

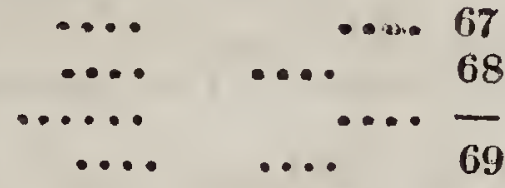
Polypody of the Oak, Rhedyn y dderwen .. Queen of the Meadow, Brenines y Weirglodd self heal, Llysiau'r Cryman Ivy, Eiddaw

Service tree, Pren Criafol

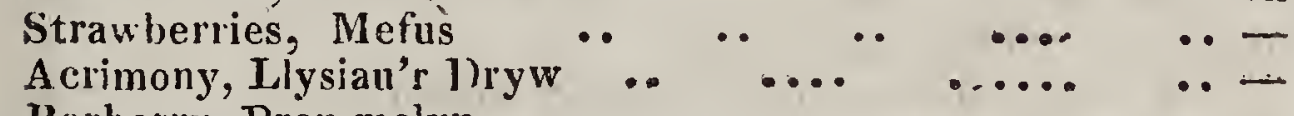
Barberry, Pren melyn $\quad$. $\quad$....... $\quad$..... $\quad$.. Small Centaury, Bustl y Ddaear .. $\quad . . \quad \ldots . . \quad$.. 73 Adder's tongue, Tafod y neidr Willow tree, Helyg

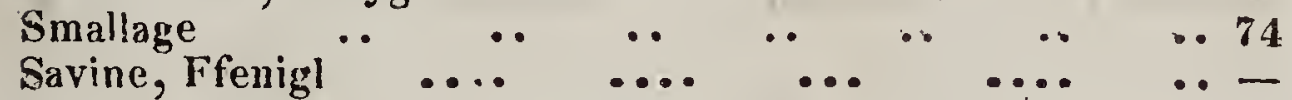

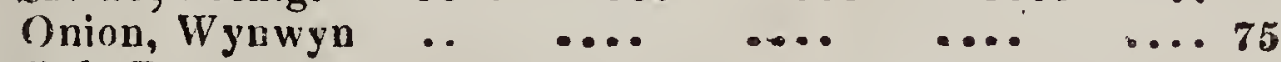
Oak, Derwen Mullein : Surcyn y melinydd Mosses, Mwsogl St. Jolin's wort, y Gantwll House leek, Llysiau pen tai .. Hops Hawthorn, Draenen wen Groundsel, y Benfelen fanyw Golden rod, y Wialen euraidd Garlick, Garlleg Fumitory, Mwsogl y ddaear Elicampane, March Alarch

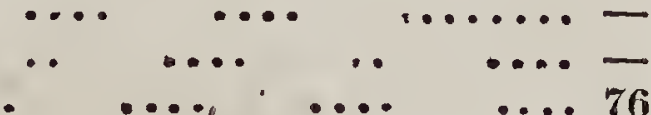
Dove's foot, y Goes goch Daises, Llygaid y dydd Crow's foot, Crafange y fran Costniary, Tafod yr Oen Hemlock, Cegyd 


\section{6}

Peony, blodau y Brenin $\quad \ldots \quad \ldots \quad \ldots \quad \ldots \quad \ldots 8$ Enwan y Planedau sydd yn rheoli llysiau, a'r amser goreu

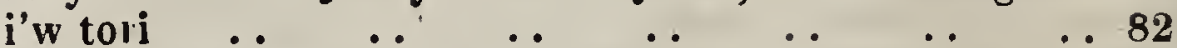

Tabl o Oriau y Planedau am bob dydd yn yr wythnos . 84

$Y$ ffordd $i$ wneuthur powltis at gasglu yr afiechyd i'r un

man, fel ag y gellir ei dori a'u sugno allan .. .. 86

Un arall i'r un dyben

Un arall

I dori chwydd coch, yn fuan

$Y$ ffordd i wneuthur Eli da i lanhau hen friwiau drewedig Cyfarwyddyd i ddefnyddio amrywiol Oilion i esmwythau

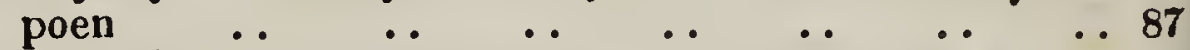

I stopio chwyydd mewn bron $\quad . \quad$.

Rhag y Clefyd melyı $\quad \ldots \quad \ldots \quad \ldots \quad$.

I stopio gwàed mewn briwiau $\quad \ldots \quad \ldots \quad \ldots \quad \ldots \quad \ldots 8$

Un arall i'r ull dyben

Cyngor i lanhau briwiau oddiwrth gig narw

Rhag y Cryd cymalau poeth, pan ag y byddoch yn methu

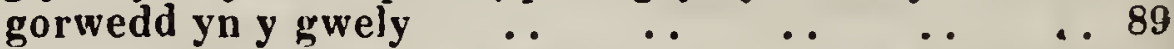

Basilicon, Eli i lanhau briwian

Eli at friwian a thyllan drewedig yn y coesau

Eli i groeni briwian

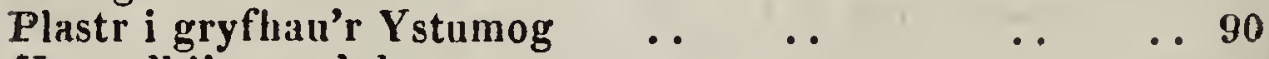

Un arall i'r un dyben

Enwau y Llysiau yn Saesoneg a Chymraeg ..

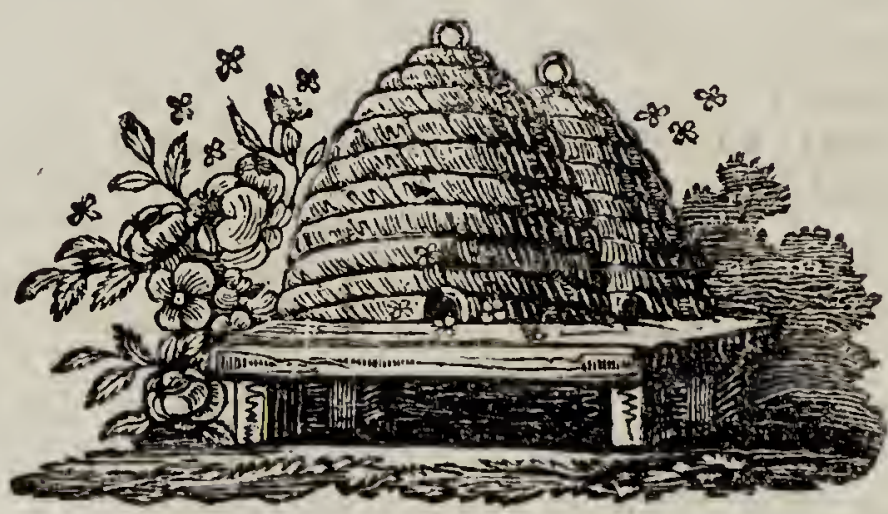

LLANRWST; ARGRAPFWYD GAN JOHY JONES: 



\section{Ilyfrau ar werth gan J.Jones, Argraffydd, Ilanrwst.}

Anianydd Cristionogol gan Dr. Dick, mewn byrddau ..........
sulianyddiaeth y sefyllfa ddyfodol, gun yr un ................... A thrawiath y ddau gyfamod, gan

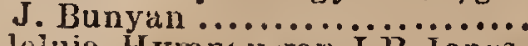
Aleiuia, Hymnau gan J.R.Jones, Ariangur wch .................. Aberth cymeradwy, gan Bunyan American, gan B. Chidlaw ...... Awengerdd Peris, Barddoniaeth, gau Gwilym Peris.............. Aduysgydd i ddarlen Cymineg Awen Lawen ................... Addysg $i$ 'r rhai bach ............... Bulim y cyfumod, gan Flavel .... Bardd Cwsg ..................... Burddoniaeth Gutyn Padaru..... Banau y byd, gun T. Edwards .. Biblau amiy w brisiau

Blodau y Gấn ...................

blwch Calliađau ................

Bl weh o Beriul .................

Bywyd Buonaparte................... By yw d Howel Harris ............... Bywyd Daniel Rowlands .......... Bywyd Dic Aberdaron............ Bywyd $T$. Edivards, Nant......... Hwrdd y Bardd, gan H. Tegai.... Bywyd $1 R$ Jones, Khoslan........ Bywyd Whitfield ............... Corph y Gaingc, gan D. Ddu, Eryr Uanialau byrion, gan y Parch S.

Roberts, Llunbrynmair ........ Cribddeiliaeth, gan W. Caledfryu Crist Datguddiedir................. Cogydd Cymueig .................... Cyiall y Cywrin ................. Cyfoeth a thlodi, gail T. E. Nant Cristion y Bibl.................... Cawell y Bara croyw............... Crist yil esiampl i'w egl wys...... Catechism J .Colquolioun, D. D. Corphyddiaeth ............... Curplı Duwinyddiaeth, gan $\ddot{A} . . .$. Patterson, IM. A............... Cymdeithas Gristionogol .......... Cronfa Awen ................... Cystrawiaeth yr Iaith Gymraeg.. Cy'farwyddwr Priodas ............ Cyfrifydd parod.................. Cyfiuwnhad, gan Dr. Owen ..... Ftto gan J. Iflias ............. Daioni a thoster Duw, gan $\dddot{\mathrm{Dr}}$. Owen ..................... Defodau $D$ wyreiniol $\ldots \ldots \ldots \ldots$ Dinystr J erusaleın ................ Drych Barddon ol gan W.Caledfryn Drych Dwfr y Cleifion ........... Drych y Ddaear a'r Fiurfafen ... Urych yr Amseroedd gan R.Jones, Rhoslan ...................... Drych $y$ Merthyron ............... Difyrwch bechgyn Glanau Conwy Deongliad bireudd wy dion 2d. a Dyitryn dewisol o fêl o'r Graig

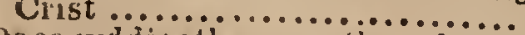
Daearyddizeth ysgrythyrol, gan

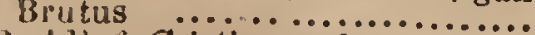
Dyddlyir Clistiono................. Disch y bydoedd ....................
Esboniad Hanesiol ar y Testament Newydd ..................... Eos Gw ynedd, gan J. Thoma....... W'wyador Peroriueth, gan H. E. $Y$ Fwyalchen ................. $Y^{r}$ Farddoneg Fabilonaidd, gan T. E. Nant .................... 0 Gardd o Gerdili gan T. Si., Nant.. I 0 Granndes Cyınıeg, gan J. Purry Gweledigaethau Nef ac Uffern, Gan J. Bunyan .............. Geirlytr yr Ysgol Subbothö.... Geirlyfr Cymiaeg a Saesneg gan y Parch. E. Griffiths ........... Geirlyfr Cymraer a Saes'lleg, gan Ellis J ones ................... Geirlyti Saesneg a Chyin raeg, gan I. $\boldsymbol{D}$. Ffraid .................. Gair o'r Gair, gan Morgan Lï H.ytorddwr yr Ymfudwr yn nghylch $W$ isconsin ............ Hyttorddw', gau y Parch T. Charles 0 Hanes Judas Iscariot ............ 00 Hanes y r Oferddy'n ............. lawn Crist, gan Jenkyn ........ \& 0 Lly thyrydd Lwyieithawg......... I 0

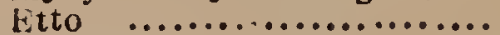
Lloches Mwyneidd-dra, gan A. $\ddot{R}$. Llais Awen..................... i 0 Mynegeir, gan y Parch P. Williams 50 Meddyg Anifeiliaid, gan David Evans, Llandyssul............ Mer Awen, sef Carolau a Cherddi Manna bob dydd ............... IMoliant o enau plant........... Myfyrdod mewn IMyn went....... Ir Oracle ..................... Pryduawngwaith y Cymry .... Pregethau Finney

Priodas ysbrydol gan J. Bunyau Pwyllwyddoriaeth (Phrenology) Pharmacopeia, (Meddyginiaeth) $Y$ Pregethwr a'r Givrandawr .... Pedai. colofn gwladwriath gan

Pregethau y $\mathrm{P}$ arch $\mathrm{D}$. Charle......

$20 \quad$ Pregethau Seryddol Dr. Chalmers

0 66 Redwar Cyflwr dyn ............

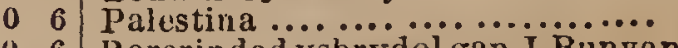

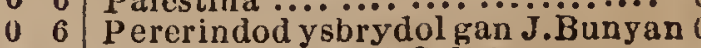

1 Rhetoreg, gan W. Salisbury .... 1

2 Rhosyn Siron, gan A. Shadrack 0

10 Rhedegwr ysprydol, gan J.Bunyan 0 Rhodd Mam i'w phlentyn........ 0 i

Sillydd Cymreig, Et to............. Testamentau Cymraeg a Saesnes, amry wiol brisiau

Telyn Arian, gan J.Thomas .... T'elyn Aur (Hymnau) ............ Tlysan Aur, gan A. Shadrack.... Bywyd Turpin Leidr ............. Tyred a chroesaw at Iesu Grist Tri chryfion byd, gan T. II. Nant Trysorfa yr Athrawon, rhwym

Do. mewn lliain................ Taith y Pererin ................. Thirza, sef Troedigaeth Iuddewes

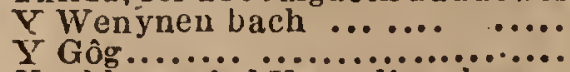
Ymddangosiud Ysprydion drwg

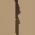

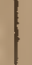

i. 